\title{
APPROXIMATION IN THE MEAN BY SOLUTIONS OF ELLIPTIC EQUATIONS
}

BY

THOMAS BAGBY

\begin{abstract}
A result analogous to the Vituškin approximation theorem is proved for mean approximation by solutions of certain elliptic equations.
\end{abstract}

1. Introduction. The theory of uniform approximation by holomorphic functions of one complex variable has a long history, including well-known results by Runge [32] and Mergeljan [28], and reached a culmination in the work of Vituškin [36]. The original proofs of these results were constructive; proofs of the results of Runge and Mergeljan by the methods of functional analysis were obtained later $[6 ; 8$, Chapter 2], but no such proof is known for the theorem of Vituškin. An analogue of the Vituškin problem for $\mathbf{L}_{p}$ approximation by holomorphic functions was considered by Havin [11], who used the methods of functional analysis and the Cartan fine topology, and by the author [3], who used the methods of functional analysis and quasitopologies. Hedberg [13] related these ideas to nonlinear potential theory, and obtained Wiener-type criteria for $\mathbf{L}_{p}$ approximation by holomorphic functions; further developments are given in the recent work of Hedberg and Wolff [18]. Lindberg [24, 25] adapted the constructive techniques of Vituškin [36] to the study of $\mathbf{L}_{p}$ approximation by holomorphic functions, obtaining, in particular, a constructive proof of the approximation theorem of [3].

The main result of the present paper is an analogue of the Vituškin theorem for $\mathbf{L}_{p}$ approximation by solutions $u$ of an equation $L(D) u \equiv L\left(\partial / \partial x_{1}, \ldots, \partial / \partial x_{n}\right) u=0$, where $L$ is a homogeneous polynomial of degree $m \geqslant 1$ in $\mathbf{R}^{n}$ with complex coefficients and

(i) $1<p<\infty$,

(ii) $L$ satisfies the ellipticity condition

$$
L(\xi) \neq 0 \quad \text { if } \xi \in \mathbf{R}^{n} \backslash\{0\},
$$

(iii) $m<n$ or $n$ is odd.

Our proof is constructive, and extends Lindberg's adaptation [25] of the method of Vituškin [36].

Generalizations of the Runge theorem to solutions of partial differential equations were given by Lax [22] and Malgrange [26]. For $p=2$, the problem considered here was studied by Babuška [2], who showed, in particular, that it was equivalent to a

Received by the editors April 20, 1981 and, in revised form, December 23, 1982.

1980 Mathematics Subject Classification. Primary 31B05, 31B15, 31B30, 35J30; Secondary 30E10. 
stability problem for the iterated Laplace operator $\Delta^{m}$ (see also Polking [30, Theorem 1.1]); the stability problem for $\Delta$ had been studied by Keldyš [21], and the stability problem for $\Delta^{s}$ has been studied more recently by Saak [33] for $s$ a positive integer satisfying $2 s<n$. For $1<p<\infty$, the problem considered here has been studied by Hedberg [14, 16, 17], Polking [30], and Hedberg and Wolff [18]. For further work on problems of the Mergeljan-Vituškin type for uniform or mean approximation by solutions of elliptic equations, see the references at the end of this paper and the references quoted in $[7 ; 12 ; 16 ; 23$, Chapter $\mathrm{V}, \S 5$ and 30].

In $\S 2$ we will define some capacities used in this paper and state our main result, Theorem 2.1. We give some auxiliary results in $\S \S 3,4$ and 5 , and then present the proof of Theorem 2.1 in $\$ \S 6$ and 7.

The author is grateful to L. I. Hedberg, who made many helpful suggestions concerning the first version of this paper. In particular, the estimate of Lemma 3.7 was originally proved by a study of Laurent-type expansions for solutions of elliptic equations (see [10]); Hedberg suggested that a more direct proof might be obtained from an application of Taylor's theorem with remainder, and this has been carried out in the final version of the paper. This improvement eliminated the need for a constant which appeared in the original statement of Theorem 2.1 and elsewhere in the paper.

Part of the work on this paper was done while the author was at Rice University, on sabbatical leave from Indiana University. The author is grateful to his home institution for making this leave possible, and to the host institution for kind hospitality and for help in the preparation of the paper.

2. Notation and statement of the approximation theorem. We begin by giving some conventions which will remain in effect for the rest of this paper.

Let $A \subset \mathbf{R}^{n}$. A neighborhood of $A$ is any open set in $\mathbf{R}^{n}$ which contains $A$. We let int $A$ denote the interior of $A$, and $\bar{A}$ the closure of $A$. We define $\chi_{A}: \mathbf{R}^{n} \rightarrow \mathbf{R}$ to be equal to one on the set $A$ and zero elsewhere.

If $\Omega \subset \mathbf{R}^{n}$ is open, we let $\Phi^{\prime}(\Omega)$ be the space of Schwartz distributions on $\Omega$, and $\mathcal{E}^{\prime}(\Omega)$ the space of distributions of compact support on $\Omega$. We use the notation $\langle T, \phi\rangle$ to denote the action of a distribution $T \in \mathscr{D}^{\prime}(\Omega)$ on a function $\phi \in C_{0}^{\infty}(\Omega)$, or the action of a distribution $T \in \mathcal{E}^{\prime}(\Omega)$ on a function $\phi \in C^{\infty}(\Omega)$.

We let $\mathbf{N}=\{0,1,2, \ldots\}$. If $\alpha=\left(\alpha_{1}, \ldots, \alpha_{n}\right) \in \mathbf{N}^{n}$, we let $|\alpha|=\alpha_{1}+\cdots+\alpha_{n}$, $\alpha !=\alpha_{1} ! \cdots \alpha_{n} !, x^{\alpha}=x_{1}^{\alpha_{1}} \cdots x_{n}^{\alpha_{n}}$ for $x=\left(x_{1}, \ldots, x_{n}\right) \in \mathbf{R}^{n}$, and $D^{\alpha}=\left(\partial / \partial x_{1}\right)^{\alpha_{1}}$ $\cdots\left(\partial / \partial x_{n}\right)^{\alpha_{n}}$. If the polynomial $P$ is given by $P(\xi)=\sum a_{\alpha} \xi^{\alpha}$, we let $\bar{P}(\xi)=\sum \overline{a_{\alpha}} \xi^{\alpha}$ and $P(D)=\Sigma a_{\alpha} D^{\alpha}$. For each $l \in \mathbf{N}$ we let $\rho_{l}$ denote the complex Hilbert space of all polynomials in $\mathbf{R}^{n}$ with complex coefficients which are homogeneous of degree $l$, with inner product [35, Chapter III, §3.1]

$$
\left\{P_{1}, P_{2}\right\}=P_{1}(D) \bar{P}_{2}=\bar{P}_{2}(D) P_{1}=\sum_{|\alpha|=l} \alpha ! c_{\alpha}^{(1)} \overline{c_{\alpha}^{(2)}},
$$

if $P_{1}$ and $P_{2}$ are elements of $\mathscr{P}_{l}$ with

$$
P_{i}(\xi)=\sum_{|\alpha|=l} c_{\alpha}^{(i)} \xi^{\alpha} \quad \text { for } \xi \in \mathbf{R}^{n} ; i=1,2 ;
$$


we let $d_{l}$ denote the dimension of $\mathscr{P}_{l}$, and we define $Y_{\alpha}(\xi)=\xi^{\alpha} / \sqrt{\alpha}$ ! for $|\alpha|=l$, so that $\left\{Y_{\alpha}\right\}_{|\alpha|=l}$ is an orthonormal basis for $\mathscr{P}_{l}$.

Throughout this paper we let $L$ be a fixed element of $\mathscr{P}_{m}, m \geqslant 1$, satisfying the ellipticity condition

$$
L(\xi) \neq 0 \quad \text { if } \xi \in \mathbf{R}^{n} \backslash\{0\}
$$

we assume that

$$
m<n \text { or } n \text { is odd. }
$$

Under these assumptions, there exists a fundamental solution $E \in \mathbf{L}_{1}\left(\mathbf{R}^{n}\right.$, loc $)$ for $L(D)$ whose restriction to $\mathbf{R}^{n} \backslash\{0\}$ is a real-analytic homogeneous function of degree $m-n$ [20, Chapter III]; we let $E$ denote a fixed fundamental solution for $L(D)$ having these properties.

If $L(D)$ is the Cauchy-Riemann operator $\partial / \partial \bar{z}=\left(\partial / \partial x_{1}+\mathbf{i} \partial / \partial x_{2}\right) / 2$ in $\mathbf{C}=\mathbf{R}^{2}$, we may take $E(z)=1 / \pi z$. If $\Delta$ is the Laplace operator $\Delta=\partial^{2} / \partial x_{1}^{2}+\cdots+\partial^{2} / \partial x_{n}^{2}$ in $\mathbf{R}^{n}, L(D)=\Delta^{m / 2}$ for some positive even integer $m$, and (2.3) holds, then we may take $E(x)=C_{n, m}|x|^{m-n}$ for some constant $C_{n, m}$.

Throughout this paper we let $p$ be a fixed real number satisfying $1<p<\infty$, and let $q$ be defined by $1 / p+1 / q=1$. The "constants" appearing in this paper may depend on $n, L, E$ and $p$, and may also depend on other quantities indicated as the constants are introduced.

If $B$ is any open ball in $\mathbf{R}^{n}$, we let $2 B$ denote the open ball with the same center as $B$ whose radius is twice that of $B$.

If $l \in\{0,1, \ldots, m-1\}$ and $H \in \mathscr{P}_{\backslash} \backslash\{0\}$, we define the capacity $\gamma_{p, H}$ as follows. Let $\Omega \subset \mathbf{R}^{n}$ be open. If $A \subset \Omega$, we set

$$
\gamma_{p, H}(A, \Omega)=\sup _{T}|\langle T, \bar{H}\rangle|,
$$

where the supremum is taken over all distributions $T \in \mathcal{E}^{\prime}\left(\mathbf{R}^{n}\right)$ such that

$$
\begin{gathered}
\operatorname{supp} T \subset A, \\
\langle T, \bar{I}\rangle=0 \quad \text { if } I \in \mathscr{P}_{0} \cup \cdots \cup \mathscr{P}_{l-1}, \\
\langle T, \bar{I}\rangle=0 \quad \text { if } I \in \mathscr{P}_{l} \text { and }\{H, I\}=0,
\end{gathered}
$$

and

$$
\|E * T\|_{p, \Omega} \leqslant 1
$$

REMARKS. 1. In view of the requirement that $T \in \mathcal{E}^{\prime}\left(\mathbf{R}^{n}\right)$, condition (2.5) means that the support of $T$ is a compact subset of $A$. In this paper we take $\|u\|_{p, A}=\|u\|_{\mathbf{L}_{p}(A)}$ if $A \subset \mathbf{R}^{n}$ is Lebesgue measurable and $u \in \mathbf{L}_{p}(A)$; and $\|u\|_{p, \Omega}=+\infty$ if $\Omega \subset \mathbf{R}^{n}$ is open and $u \in \mathscr{Q}^{\prime}(\Omega) \backslash \mathbf{L}_{p}(\Omega)$. Note that if $A$ is a subset of the open set $\Omega \subset \mathbf{R}^{n}$, and $T \in \mathcal{E}^{\prime}\left(\mathbf{R}^{n}\right)$ satisfies (2.5) and (2.8), then $E * T \in \mathbf{L}_{p}\left(\mathbf{R}^{n}\right.$, loc $)$.

2. We use the convention that if $l=0$, the symbol $\mathscr{P}_{0} \cup \cdots \cup \mathscr{P}_{l-1}$ refers to the empty set. Thus, in the special case $H \in \mathscr{P}_{0} \backslash\{0\}$, condition (2.6) may be omitted, and, since $\operatorname{dim}_{\mathbf{C}} \mathscr{P}_{0}=1$, condition (2.7) may also be omitted. 
3. The capacity $\gamma_{p, 1}$ was studied by Harvey and Polking [9], and is related to the capacities defined in nonlinear potential theory (see the discussion following Theorem 2.1 below). The capacities $\gamma_{p, H}$ are similar to a family of capacities defined by Maz'ja [27]. See also Hedberg [15] and the references given there.

We give an alternate definition for $\gamma_{p . H}$ in the appendix; the discussion there provides motivation for the definition above.

THeOREM 2.1. Let $X \subset \mathbf{R}^{n}$ be a compact set of positive Lebesgue measure. Then the following conditions are equivalent:

(a) If $f \in \mathbf{L}_{p}(X)$, and $L(D) f=0$ on int $X$, then for each $\varepsilon>0$ there exists $a$ solution $u$ of $L(D) u=0$ on a neighborhood of $X$ such that $\|u-f\|_{p, X}<\varepsilon$.

(b) If $H \in \mathscr{P}_{0} \cup \cdots \cup \mathscr{P}_{m-1} \backslash\{0\}$, and if $G$ and $\Omega$ are open subsets of $\mathbf{R}^{n}$ satisfying $G \Subset \Omega \Subset \mathbf{R}^{n}$, then

$$
\gamma_{p, H}(G \backslash \text { int } X, \Omega)=\gamma_{p, H}(G \backslash X, \Omega) .
$$

(c) There exist $\eta>0$ and $\rho>0$ with the following property: if $H \in \mathscr{P}_{0}$ $\cup \cdots \cup \mathscr{P}_{m-1} \backslash\{0\}$, and $B$ is an open ball in $\mathbf{R}^{n}$ with radius less than $\rho$, then

$$
\gamma_{p, H}(B \backslash \text { int } X, 2 B) \leqslant \eta \gamma_{p, H}(B \backslash X, 2 B) .
$$

A theorem of Polking states that (with slightly different definitions) condition (b) for the case $H \in \mathcal{P}_{0} \backslash\{0\}$ follows from (a), and implies that condition (c) for the case $H \in \mathscr{P}_{0} \backslash\{0\}$ follows from (a). Polking's result is quoted in Remark 5 below.

In case $q>n$, it follows easily from Remarks 1 and 2 in $\S 4$ and Propositions 4.2, 4.5 and 4.6 that condition (c) of Theorem 2.1 holds for every compact set $X \subset \mathbf{R}^{n}$. We conclude that if $q>n$, condition (a) of Theorem 2.1 must hold for every compact set $X \subset \mathbf{R}^{n}$; this was proved by Polking [30, Theorem 2.9], and related results were proved by Burenkov [4].

For the operator $L(D)=\partial / \partial \bar{z}$ in $\mathbf{R}^{2}$, Theorem 2.1 is known, and, in fact, a number of other equivalent conditions have been given; for further discussion and references see the first paragraph of $\$ 1$.

In order to place Theorem 2.1 in perspective, we will conclude this section with several remarks concerning earlier work on the use of capacities to study mean approximation by solutions of elliptic equations. For this purpose we let $P(D)$ be any elliptic partial differential operator of order $k \geqslant 1$ in $\mathbf{R}^{n}$ having constant coefficients. Let $X \subset \mathbf{R}^{n}$ be compact. We say that $X$ has the $\mathbf{L}_{p}$ approximation property for $P(D)$ provided condition (a) of Theorem 2.1 holds with $P(D)$ in place of $L(D)$. If $-\infty<s<\infty$, we define the Bessel kernel $G_{s}$ by requiring that the Fourier transform $\hat{G}_{s}(\xi)=\left(1+|\xi|^{2}\right)^{-s / 2}$, and for $A \subset \mathbf{R}^{n}$ we define the capacity $c_{s, q}(A)=$ $\sup |\langle T, 1\rangle|$, where the supremum is taken over all $T \in \mathcal{E}^{\prime}\left(\mathbf{R}^{n}\right)$ such that $\operatorname{supp} T \subset A$ and $\left\|G_{s} * T\right\|_{p, \mathbf{R}^{n}} \leqslant 1$. The theory of the capacities $c_{s, q}$ and the related nonlinear potentials has been developed by Adams, Fuglede, Havin, Hedberg, Maz'ja, Meyers, Rešetnjak and others; for further discussion, and comparison with other capacities used in nonlinear potential theory, see $[1,16,17,30]$ and the references given there. In the following remarks we use the equivalent formulations of the $\mathbf{L}_{p}$ approximation property proved by Polking [30, Theorem 1.1], as well as basic properties of the capacities $c_{s, q}$, for which a convenient reference is $[30, \S 2]$. 
Remarks. 4. Polking [30] and Hedberg [14, 16, 17] have proved sufficient conditions for the $\mathbf{L}_{p}$ approximation property. In particular, the work of Hedberg [17] on the spectral synthesis problem for Sobolev spaces, as extended by Hedberg and Wolff [18], yields the following result. If there exists a constant $\eta>0$ such that

$$
c_{i, q}(G \backslash \operatorname{int} X) \leqslant \eta c_{i, q}(G \backslash X)
$$

for all open sets $G \subset \mathbf{R}^{n}$ and each $i=1, \ldots, k$, then $X$ has the $\mathbf{L}_{p}$ approximation property for $P(D)$.

5. Polking [30, Theorem 2.7] proved the following necessary condition for mean approximation. If $X$ has the $\mathbf{L}_{p}$ approximation property for $P(D)$, then $c_{k, q}(G \backslash$ int $X)$ $=c_{k, q}(G \backslash X)$ for every open set $G \subset \mathbf{R}^{n}$. Polking has pointed out to the author that it is not known whether the converse is true in general (compare [33]).

6. For $k \geqslant 2$ there is a gap between the conditions given by the italicized statements in Remarks 4 and 5. In this connection, Hedberg has pointed out the following two illuminating examples to the author:

(a) The converse of the italicized statement in Remark 4 is false if $n / k<q \leqslant n$. To prove this, note that since $q \leqslant n$, it follows from [31, Theorem 4 and 30, Theorem $\left.2.6, c_{2}\right) \Rightarrow$ a)] that there exists a nowhere dense compact set $X \subset \mathbf{R}^{n}$ such that for any $\eta>0$, condition (2.9) for $i=1$ will fail for some open set $G \subset \mathbf{R}^{n}$. However, since $X$ is nowhere dense and $n / k<q$, it follows from [30, Theorem 2.6] that $X$ has the $\mathbf{L}_{p}$ approximation property for $P(D)$.

(b) Although we do not know whether the converse to the italicized statement in Remark 5 is valid, we can show the following: if $k \geqslant 2$ and $q=n$, then there exists a compact set $X \subset \mathbf{R}^{n}$ and a constant $\eta$ such that (2.9) holds for $i=k$ and every open set $G \subset \mathbf{R}^{n}$ of diameter less than 1 , but $X$ does not have the $\mathbf{L}_{p}$ approximation property for $P(D)$. To prove this, note that since $q=n$, it follows from [16, Example 6.6] that there exists a compact set $X \subset \mathbf{R}^{n}$ which does not have the approximation property for $P(D)$. Since $k q>n$, the set $\left\{c_{k, q}(A): A \subset \mathbf{R}^{n}, A\right.$ nonempty $\}$ has a positive lower bound; it follows that (2.9) holds for $i=k$ and every open set $G \subset \mathbf{R}^{n}$ of diameter less than 1 .

7. The work of Saak [33] gives conditions involving certain capacities which are both necessary and sufficient for a compact set $X \subset \mathbf{R}^{n}$ to have the $\mathbf{L}_{2}$ approximation property for $P(D)$, provided $2 k<n$.

3. Preliminary results. If $a \in \mathbf{R}^{n}$ and $r>0$, we let $\mathbf{B}_{r}(a)=\left\{x \in \mathbf{R}^{n}:|x-a|<r\right\}$ be the open ball of radius $r$ about $a$, and we write $\mathbf{B}_{r}=\mathbf{B}_{r}(0)$. We let $\lambda$ denote Lebesgue measure in $\mathbf{R}^{n}$, and $\boldsymbol{\delta}$ the Dirac measure at the origin of $\mathbf{R}^{n}$. If $f \in$ $\mathbf{L}_{1}\left(\mathbf{R}^{n}\right.$, loc $)$, and $A \subset \mathbf{R}^{n}$ is a bounded Lebesgue measurable set with $\lambda(A)>0$, we set

$$
[f]_{A}=\frac{1}{\lambda(A)} \int_{A} f d \lambda .
$$

We will use the elementary estimate

$$
\left|\sum_{j=1}^{t} z_{j}\right|^{p} \leqslant t^{p / q} \sum_{j=1}^{t}\left|z_{j}\right|^{p}
$$


for any complex numbers $z_{1}, \ldots, z_{t}$. This follows from Hölder's inequality for finite sums.

We now give some results concerning "potentials" given by the convolution of the fundamental solution $E$ and distributions of compact support in $\mathbf{R}^{n}$.

Lemma 3.1. Let $T \in \mathcal{E}^{\prime}\left(\mathbf{R}^{n}\right)$. If the distribution $E * T$ coincides with the zero distribution on a neighborhood of $\operatorname{supp} T$, then $T=0$.

Proof. Since $T=\delta * T=L(D)(E * T)$, the hypothesis implies that $T$ coincides with the zero distribution on a neighborhood of $\operatorname{supp} T$. Thus $T=0$.

Lemma 3.2. Let $\psi \in C^{\infty}\left(\mathbf{R}^{n}\right)$. Let $K$ be a compact subset of the open set $\Omega \subset \mathbf{R}^{n}$, and let $\phi \in C_{0}^{\infty}(\Omega)$ be identically equal to one on a neighborhood of $K$. If $T \in \mathcal{E}^{\prime}\left(\mathbf{R}^{n}\right)$ satisfies supp $T \subset K$, then

$$
|\langle T, \psi\rangle| \leqslant\|E * T\|_{p, \Omega}\|L(D)(\phi \psi)\|_{q, \Omega} .
$$

Proof. If $T \in \mathcal{E}^{\prime}\left(\mathbf{R}^{n}\right)$ satisfies supp $T \subset K$, then we obtain, from Hölder's inequality,

$$
\begin{aligned}
|\langle T, \psi\rangle| & =|\langle T, \phi \psi\rangle|=|\langle L(D)(E * T), \phi \psi\rangle| \\
& =|\langle E * T, L(D)(\phi \psi)\rangle| \leqslant\|E * T\|_{p, \Omega}\|L(D)(\phi \psi)\|_{q, \Omega} .
\end{aligned}
$$

Corollary 3.3. Let $l \in \mathbf{N}$, and let $K$ be a compact subset of the open set $\Omega \subset \mathbf{R}^{n}$. Then there exists a constant $C=C(l, K, \Omega)$ such that, for any distribution $T \in \mathcal{E}^{\prime}\left(\mathbf{R}^{n}\right)$ satisfying supp $T \subset K$, we have

$$
|\langle T, I\rangle| \leqslant C\{I, I\}^{1 / 2}\|E * T\|_{p, \Omega} \quad \text { if } I \in \mathscr{P}_{0} \cup \cdots \cup \mathscr{P}_{l} .
$$

Proof. Let $\phi \in C_{0}^{\infty}(\Omega)$ be identically equal to one on a neighborhood of $K$, and let $T \in \mathcal{E}^{\prime}\left(\mathbf{R}^{n}\right)$ satisfy supp $T \subset K$. If $|\alpha| \leqslant l$, then from Lemma 3.2 we have

$$
\left|\left\langle T, Y_{\alpha}\right\rangle\right| \leqslant\|E * T\|_{p, \Omega}\left\|L(D)\left(\phi Y_{\alpha}\right)\right\|_{q, \Omega} .
$$

If $I \in \mathscr{P}_{k}$, where $\mathbf{N} \ni k \leqslant l$, we may write $I=\Sigma_{|\alpha|=k} c_{\alpha} Y_{\alpha}$, for some choice of complex numbers $c_{\alpha}$; then $\Sigma_{|\alpha|=k}\left|c_{\alpha}\right| \leqslant d_{k}^{1 / 2}\{I, I\}^{1 / 2}$, and hence (3.4) yields (3.3) with $C=d_{k}^{1 / 2} \sup _{|\alpha| \leqslant l}\left\|L(D)\left(\phi Y_{\alpha}\right)\right\|_{q, \Omega}$.

LEMMA 3.4. There exists a constant $C$ such that for every $P \in \mathscr{P}_{m}$ we have

$$
\|P(D)(E * f)\|_{p, \mathbf{R}^{n}} \leqslant C\{P, P\}^{1 / 2}\|f\|_{p, \mathbf{R}^{n}}
$$

if $f \in \mathbf{L}_{p}\left(\mathbf{R}^{n}\right)$ has compact support.

Proof. We first let $\alpha \in \mathbf{N}^{n}$ satisfy $|\alpha|=m$. Then $Y_{\alpha}(D) E$ is a tempered distribution on $\mathbf{R}^{n}$ whose restriction to $\mathbf{R}^{n} \backslash\{0\}$ is infinitely differentiable and homogeneous of degree $-n$. We conclude from $[5, \S 3.4$, Theorem 26] that the Fourier transform $\left(Y_{\alpha}(D) E\right)^{\wedge}$ is an element of $\mathbf{L}_{1}\left(\mathbf{R}^{n}\right.$, loc $)$. From the equation $\left[L(D) Y_{\alpha}(D) E\right]^{\hat{}}=$ $\left[Y_{\alpha}(D) \delta\right]^{\hat{n}}$, and the fact that $L$ satisfies the ellipticity condition (2.2), we see that the restriction of $\left(Y_{\alpha}(D) E\right)^{\wedge}$ to $\mathbf{R}^{n} \backslash\{0\}$ is infinitely differentiable and homogeneous of degree 0 (in fact, $\left(Y_{\alpha}(D) E\right)^{\wedge}$ is a constant multiple of the function $\xi \rightarrow \xi^{\alpha} / L(\xi)$ ). It then follows from the theory of singular integrals of Mihlin, Calderón and Zygmund, 
and Hörmander that there exists a constant $C_{\alpha}$ such that

$$
\left\|Y_{\alpha}(D)(E * f)\right\|_{p, \mathbf{R}^{n}} \leqslant C_{\alpha}\|f\|_{p, \mathbf{R}^{n}}
$$

for all functions $f \in \mathbf{L}_{p}\left(\mathbf{R}^{n}\right)$ having compact support. (See [35, Chapter III, §3.5 or 35, Chapter IV, §3.2].)

If $P \in \mathscr{P}_{m}$, we may write $P=\sum_{|\alpha|=m} c_{\alpha} Y_{\alpha}$, for some choice of complex numbers $c_{\alpha}$; then

$$
\sum_{|\alpha|=m}\left|c_{\alpha}\right| \leqslant d_{m}^{1 / 2}\{P, P\}^{1 / 2}
$$

and hence (3.6) yields (3.5) with $C=d_{m}^{1 / 2} \sup _{|\alpha|=m} C_{\alpha}$.

We conclude this section with a "decay estimate for potentials", stated as Lemma 3.7. For $L(D)=\partial / \partial \bar{z}$ and $E(z)=1 / \pi z$, such estimates are known and have been used in the study of approximation by holomorphic functions $[24,25,28,36]$.

The following lemma is known (see [34, §3.4.2]), but for completeness we include the proof.

LEMMA 3.5. Let $K$ be a compact subset of the open set $\Omega \subset \mathbf{R}^{n}$. Then for each $\alpha \in \mathbf{N}^{n}$ there exists a constant $C=C(\Omega, K, \alpha)$ with the following property. If $u \in$ $\mathbf{L}_{p}(\Omega)$ satisfies $L(D) u=0$ in $\Omega$, then

$$
\left|D^{\alpha} u(x)\right| \leqslant C\|u\|_{p, \Omega} \quad \text { for } x \in K .
$$

Proof. Let $0<d<\operatorname{dist}\left(K, \mathbf{R}^{n} \backslash \Omega\right) \leqslant \infty$, and let $\psi \in C_{0}^{\infty}\left(\mathbf{B}_{d / 2}\right)$ satisfy $\psi \equiv 1$ in a neighborhood of the origin. Let $\alpha \in \mathbf{N}^{n}$. If $u \in \mathbf{L}_{p}(\Omega)$ satisfies $L(D) u=0$ in $\Omega$, we may regard $u \in \mathbf{L}_{p}\left(\mathbf{R}^{n}\right) \subset \mathscr{Q}^{\prime}\left(\mathbf{R}^{n}\right)$ by setting $u \equiv 0$ in $\mathbf{R}^{n} \backslash \Omega$. We then have

$$
\begin{aligned}
\operatorname{supp}\left[D^{\alpha} L(D)(\psi E)\right] * u & =\operatorname{supp}\left[D^{\alpha}(\psi E)\right] *[L(D) u] \\
& \subset \operatorname{supp} D^{\alpha}(\psi E)+\operatorname{supp} L(D) u \subset \mathbf{B}_{d / 2}+\left(\mathbf{R}^{n} \backslash \Omega\right) .
\end{aligned}
$$

It follows that on the set $K+\mathbf{B}_{d / 2}$ we have

$$
\left\{D^{\alpha} L(D)[(1-\psi) E]\right\} * u=\left\{D^{\alpha} L(D) E\right\} * u=D^{\alpha} u
$$

In particular, for each $x \in K$ we obtain

$$
\begin{aligned}
\left|D^{\alpha} u(x)\right| & =\left|\int_{\mathbf{B}_{d / 2}} u(x-y) D^{\alpha} L(D)[(1-\psi(y)) E(y)] d \lambda(y)\right| \\
& \leqslant \sup _{\mathbf{R}^{n}}\left\{D^{\alpha} L(D)[(1-\psi) E]\right\} \int_{K+\mathbf{B}_{d / 2}}|u| d \lambda
\end{aligned}
$$

the lemma follows from applying Hölder's inequality to the last integral.

For each $x \in \mathbf{R}^{n} \backslash\{0\}$ and each $l \in \mathbf{N}$ we define the polynomial

$$
Q_{l}^{(x)}(y)=(-1)^{l} \sum_{|\alpha|=l}\left[D^{\alpha} E(x)\right] y^{\alpha} / \alpha !
$$


If $x \in \mathbf{R}^{n} \backslash\{0\}$ is fixed, the function $\mathbf{R}^{n} \backslash\{x\} \ni y \rightarrow E(x-y) \in \mathbf{C}$ is real-analytic; hence the Taylor expansion about $y=0$,

$$
E(x-y)=\sum_{l=0}^{\infty} Q_{l}^{(x)}(y),
$$

must be valid for $y$ in some neighborhood of the origin in $\mathbf{R}^{n}$.

LEMMA 3.6. Let $l \in \mathbf{N}$.

(a) There exists a constant $C=C(l)$ such that $\left|Q_{l}^{(x)}(y)\right| \leqslant C|x|^{m-n-l}|y|^{l}$ if $x \in$ $\mathbf{R}^{n} \backslash\{0\}$ and $y \in \mathbf{R}^{n}$.

(b) If $x \in \mathbf{R}^{n} \backslash\{0\}$ is fixed, then $L(D) Q_{l}^{(x)}=0$ in $\mathbf{R}^{n}$.

Proof. Part (a) follows from the fact that for each $\alpha \in \mathbf{N}^{n}, D^{\alpha} E$ is homogeneous of degree $m-n-|\alpha|$.

To prove part (b) we note that the conclusion is obvious if $l<m$, and hence we assume that $l \geqslant m$. In the Taylor expansion (3.7) we may apply $L(D)$ to each monomial, and then combine terms to obtain the Taylor series for the function

$$
\mathbf{R}^{n} \backslash\{x\} \ni y \rightarrow L(D) E(x-y) \equiv 0
$$

near $y=0$; in the latter Taylor series each monomial must have coefficient zero, and it follows that $L(D) Q_{l}^{(x)}=0$ near $y=0$. Since $Q_{l}^{(x)} \in \mathscr{P}_{l}$, the lemma follows.

Lemma 3.7. For each $l_{0} \in \mathbf{N}$ there exists a constant $C=C\left(l_{0}\right)$ with the following property. Suppose that $T \in \mathcal{G}^{\prime}\left(\mathbf{R}^{n}\right)$ satisfies $\operatorname{supp} T \subset \overline{\mathbf{B}}_{1}$, and if $l_{0} \geqslant 1$ suppose that

$$
\langle T, I\rangle=0 \text { for } I \in \mathscr{P}_{0} \cup \cdots \cup \mathscr{P}_{I_{0}-1} \text {. }
$$

If $u=E * T \in \mathbf{L}_{p}\left(\mathbf{R}^{n}\right.$, loc $)$, then

$$
|u(x)| \leqslant C\|u\|_{p, \mathbf{B}_{2} \backslash \mathbf{B}_{1}}|x|^{m-n-l_{0}} \text { for }|x| \geqslant 2 .
$$

Proof. Let $\phi \in C_{0}^{\infty}\left(\mathbf{B}_{5 / 3}\right)$ be a fixed function which is identically equal to one on a neighborhood of $\overline{\mathbf{B}_{4 / 3}}$. Now suppose that $T \in \mathcal{E}^{\prime}\left(\mathbf{R}^{n}\right)$ and $u=E * T$ satisfy the hypotheses of the lemma, define $\tilde{u}=(1-\phi) u$ and make the following three observations:

$1^{\circ} . \hat{u}=E * L(D) \tilde{u}$.

To prove $1^{\circ}$, we first note that $u-\tilde{u}$ has compact support, and hence

$$
\begin{aligned}
u-\tilde{u} & =\boldsymbol{\delta} *(u-\tilde{u})=L(D)(E *(u-\tilde{u})) \\
& =E * L(D)(u-\tilde{u}) .
\end{aligned}
$$

Since we also have $u=E * T=E *(L(D) u), 1^{\circ}$ follows.

$2^{\circ}$. If $Q \in \mathscr{P}_{0} \cup \cdots \cup \mathscr{P}_{l_{0}-1}$ satisfies $L(D) Q=0$, then $\langle L(D) \tilde{u}, Q\rangle=0$.

In fact, since $u-\tilde{u}$ has compact support, we have

$$
\langle L(D)(u-\tilde{u}), Q\rangle=(-1)^{m}\langle u-\tilde{u}, L(D) Q\rangle=0 .
$$

Since we also have $\langle L(D) u, Q\rangle=0$ by $(3.8), 2^{\circ}$ follows.

$3^{\circ}$. There is a constant $C_{1}$, depending on $\phi$, such that $\sup _{\mathbf{R}^{n}}|L(D) \tilde{u}| \leqslant$ $C_{1}\|u\|_{p, \mathbf{B}_{2} \backslash \overline{\mathbf{B}}_{1}}$. 
To prove $3^{\circ}$, note that we may write

$$
L(D) \tilde{u}(y)=\sum_{|\alpha+\beta|=m} c_{\alpha \beta}\left(D^{\alpha} \phi(y)\right)\left(D^{\beta} u(y)\right)
$$

for some choice of complex numbers $c_{\alpha \beta}$ depending only on $L(D)$. Thus $3^{\circ}$ follows from Lemma 3.5 when $K=\overline{\mathbf{B}_{5 / 3}} \backslash \mathbf{B}_{4 / 3}$ and $\Omega=\mathbf{B}_{2} \backslash \overline{\mathbf{B}_{1}}$.

Now fix $x \in \mathbf{R}^{n}$ with $|x| \geqslant 2$. If $y \in \overline{\mathbf{B}_{5 / 3}}$, it follows from Taylor's theorem with remainder that

$$
E(x-y)=\sum_{l=0}^{l_{0}-1} Q_{l}^{(x)}(y)+Q_{l_{0}}^{(x-\tilde{y})}(y),
$$

where $\tilde{y}$ is some point on the segment from 0 to $y$ (if $l_{0}=0$, the summation term is omitted); by Lemma 3.6(a) we have

$$
\left|Q_{l_{0}}^{(x-\tilde{y})}(y)\right| \leqslant C_{2}|x-\tilde{y}|^{m-n-l_{0}}|y|^{l_{0}} \leqslant C_{3}|x|^{m-n-l_{0}}|y|^{l_{0}},
$$

where $C_{2}$ is the constant $C\left(l_{0}\right)$ of Lemma 3.6(a) and $C_{3}=6^{\left|m-n-l_{0}\right|} C_{2}$. We therefore conclude from $1^{\circ}, 2^{\circ}$ and Lemma 3.6(b) that

$$
\begin{aligned}
|u(x)| & =|E * L(D) \tilde{u}(x)|=\left|\int E(x-y) L(D) \tilde{u}(y) d \lambda(y)\right| \\
& \leqslant(5 / 3)^{l_{0}} C_{3} \lambda\left(\mathbf{B}_{5 / 3}\right)|x|^{m-n-l_{0}} \sup _{\mathbf{R}^{n}}|L(D) \tilde{u}| .
\end{aligned}
$$

From this estimate and $3^{\circ}$ we obtain (3.9).

4. The capacities $\gamma_{p, H}$. In this section we study the capacities $\gamma_{p, H}$. Throughout this section we let $l \in\{0,1, \ldots, m-1\}$ be fixed. We first let $H \in \mathscr{P}_{\perp} \backslash\{0\}$, and make the following observations.

REMARKS. 1. If $K$ is a compact subset of the open set $\Omega \subset \mathbf{R}^{n}$, then $\gamma_{p, H}(K, \Omega) \leqslant$ $C\{H, H\}^{1 / 2}$, where $C=C(l, K, \Omega)$ is the constant of Corollary 3.3.

2. If $\Omega$ and $\Omega_{0}$ are open sets in $\mathbf{R}^{n}$, then

$$
\begin{array}{cc}
\gamma_{p, H}\left(A_{1}, \Omega\right) \leqslant \gamma_{p, H}\left(A_{2}, \Omega\right) & \text { for } A_{1} \subset A_{2} \subset \Omega, \\
\gamma_{p, H}(A, \Omega) \leqslant \gamma_{p, H}\left(A, \Omega_{0}\right) & \text { for } A \subset \Omega_{0} \subset \Omega .
\end{array}
$$

3. If $A$ is a subset of the open set $\Omega \subset \mathbf{R}^{n}$, and $\gamma_{p, H}(A, \Omega)>0$, then

$$
\gamma_{p, H}(A, \Omega)=\sup _{T}|\langle T, \bar{H}\rangle|,
$$

where the supremum is taken over all distributions $T \in \mathcal{E}^{\prime}\left(\mathbf{R}^{n}\right)$ satisfying (2.5)-(2.8) and $0<\|E * T\|_{p, \Omega}$.

If $\phi \in C^{\infty}\left(\mathbf{R}^{n}\right)$ and $a \in \mathbf{R}^{n}$, we define the translated function $\phi_{a} \in C^{\infty}\left(\mathbf{R}^{n}\right)$ by $\phi_{a}(x)=\phi(x-a), x \in \mathbf{R}^{n} ;$ if $u \in \mathscr{D}^{\prime}\left(\mathbf{R}^{n}\right)$ and $a \in \mathbf{R}^{n}$, we define the translated distribution $u_{a} \in \mathscr{D}^{\prime}\left(\mathbf{R}^{n}\right)$ by $\left\langle u_{a}, \phi\right\rangle=\left\langle u, \phi_{-a}\right\rangle, \phi \in C_{0}^{\infty}\left(\mathbf{R}^{n}\right)$, and note that supp $u_{a}$ $=[\operatorname{supp} u]+\{a\}$. 
LemmA 4.1. Let $k \in \mathbf{N}, T \in \mathcal{E}^{\prime}\left(\mathbf{R}^{n}\right)$, and $a \in \mathbf{R}^{n}$. Suppose that $\langle T, I\rangle=0$ if $I \in \mathscr{P}_{0} \cup \cdots \cup \mathscr{P}_{k}$. Then $\left\langle T, I_{a}\right\rangle=\langle T, I\rangle$ if $I \in \mathscr{P}_{k+1}$.

Proof. If $I \in \mathscr{P}_{k+1}$, we may write $I_{a}=\sum_{j=0}^{k+1} P_{j}$, where $P_{k+1}=I$ and $P_{j} \in \mathscr{P}_{j}$ for $0 \leqslant j \leqslant k$. The lemma follows.

From Lemma 4.1 we obtain the invariance of the capacities $\gamma_{p, H}$ under translation of $\mathbf{R}^{n}$ :

Proposition 4.2. Let $H \in \mathscr{P}_{\Lambda} \backslash\{0\}$. If $\Omega \subset \mathbf{R}^{n}$ is open, $A \subset \Omega$, and $a \in \mathbf{R}^{n}$, then $\gamma_{p, H}(A, \Omega)=\gamma_{p, H}(A+\{a\}, \Omega+\{a\})$.

We now discuss the behavior of the capacities $\gamma_{p, H}$ under the mapping $\mathcal{T}: \mathbf{R}^{n} \rightarrow \mathbf{R}^{n}$ defined by

$$
\mathcal{T}(x)=\delta x \text { if } x \in \mathbf{R}^{n} \text {, }
$$

where $\delta>0$ is fixed. Under the diffeomorphism (4.3), each function $f \in \mathbf{L}_{1}\left(\mathbf{R}^{n}\right.$, loc) defines a "push-forward" function $\tilde{f}=f \circ \mathcal{T}^{-1} \in \mathbf{L}_{1}\left(\mathbf{R}^{n}\right.$, loc $)$. If $f \in \mathbf{L}_{p}\left(\mathbf{R}^{n}\right.$, loc $)$, the change-of-variables formula gives $\tilde{f} \in \mathbf{L}_{p}\left(\mathbf{R}^{n}\right.$, loc $)$ and, in fact,

$$
\|\tilde{f}\|_{p, \tau(\Omega)}=\delta^{n / p}\|f\|_{p . \Omega}
$$

for each open set $\Omega \subset \mathbf{R}^{n}$.

Under the diffeomorphism (4.3), each distribution $u \in \mathscr{D}^{\prime}\left(\mathbf{R}^{n}\right)$ defines a "pushforward" distribution $\tilde{u} \in \mathscr{Q}^{\prime}\left(\mathbf{R}^{n}\right)$ by the rule $[19, \S 1.8]$

$$
\tilde{u}(\phi)=\delta^{n} u(\phi \circ \mathcal{T}) \quad \text { if } \phi \in C_{0}^{\infty}\left(\mathbf{R}^{n}\right) .
$$

The change-of-variables formula shows that if $u \in \mathscr{D}^{\prime}\left(\mathbf{R}^{n}\right)$ is the distribution defined by the function $f \in \mathbf{L}_{1}\left(\mathbf{R}^{n}\right.$, loc), then the push-forward $\tilde{u} \in \mathscr{D}^{\prime}\left(\mathbf{R}^{n}\right)$ is the distribution defined by the push-forward $\tilde{f} \in \mathbf{L}_{1}\left(\mathbf{R}^{n}\right.$, loc $)$. If $u \in \mathscr{Q}^{\prime}\left(\mathbf{R}^{n}\right)$, we have

$$
\operatorname{supp} \tilde{u}=\mathcal{T}(\operatorname{supp} u) \text {. }
$$

The proofs of the next two lemmas are routine and will be omitted.

Lemma 4.3. If $k \in \mathbf{N}$ and $T \in \mathcal{E}^{\prime}\left(\mathbf{R}^{n}\right)$, then $\langle\tilde{T}, I\rangle=\delta^{n+k}\langle T, I\rangle$ for $I \in \mathscr{P}_{k}$.

LEMMA 4.4. If $T \in \mathcal{E}^{\prime}\left(\mathbf{R}^{n}\right)$, then $[E * T]^{\tilde{}}=\delta^{-m} E * \tilde{T}$.

Proposition 4.5. Let $H \in \mathscr{P}_{\backslash} \backslash\{0\}$. If $\Omega \subset \mathbf{R}^{n}$ is open, and $A \subset \Omega$, then

$$
\gamma_{p, H}(\mathcal{T}(A), \mathcal{T}(\Omega))=\delta^{-m+l+n / q} \gamma_{p, H}(A, \Omega) \text {. }
$$

Proof. Let $T \in \mathcal{E}^{\prime}\left(\mathbf{R}^{n}\right)$ satisfy (2.5)-(2.8). Using (4.4), (4.5) and Lemmas 4.3 and 4.4 , we conclude that $\gamma_{p . H}(\widetilde{T}(A), \widetilde{T}(\Omega)) \geqslant \delta^{-m-n / p}|\langle\tilde{T}, \bar{H}\rangle|$, and from Lemma 4.3 we then obtain

$$
\gamma_{p, H}(\mathcal{J}(A), \mathcal{T}(\Omega)) \geqslant \delta^{-m+l+n / q}|\langle T, \bar{H}\rangle| .
$$

We have thus proved that

$$
\gamma_{p, H}(\widetilde{J}(A), \mathcal{J}(\Omega)) \geqslant \delta^{-m+l+n / q} \gamma_{p, H}(A, \Omega),
$$

and by applying the same reasoning to the mapping $\widetilde{T}^{-1}$ we obtain Proposition 4.5. 
Proposition 4.6. Let $\Omega$ be a bounded open set in $\mathbf{R}^{n}$.

(a) If $q(m-l) \leqslant n$ and $H \in \mathscr{P}_{l} \backslash\{0\}$, then $\gamma_{p, H}(\{a\}, \Omega)=0$ for $a \in \Omega$.

(b) If $q(m-l)>n$, then there exists a constant $C=C(l, \Omega)>0$ such that for each $H \in \mathscr{P}_{l} \backslash\{0\}$ we have $\gamma_{p, H}(\{a\}, \Omega) \geqslant C\{H, H\}^{1 / 2}$ if $a \in \Omega$.

Proof. (a) In view of Proposition 4.2, we may assume that $a=0$. We may then write $\mathbf{B}_{r} \subset \Omega$ for some $r>0$; in view of the monotonicity property (4.2), it suffices to prove

$$
\gamma_{p, H}\left(\{0\}, \mathbf{B}_{r}\right)=0 \text { for } r>0,
$$

and we distinguish two cases.

Case 1. $q(m-l)<n$. If $\gamma_{p, H}\left(\{0\}, \mathbf{B}_{R}\right)>0$ for some $R>0$, it follows from Proposition 4.5 and the hypothesis $q(m-l)<n$ that the function $(0, \infty) \ni r \rightarrow$ $\gamma_{p, H}\left(\{0\}, \mathbf{B}_{r}\right)$ is strictly increasing. This contradicts the monotonicity property (4.2), and hence (4.6) must hold.

Case 2. $q(m-l)=n$. If $\gamma_{p, H}\left(\{0\}, \mathbf{B}_{R}\right)>0$ for some $R>0$, then we can find a distribution $T \in \mathcal{E}^{\prime}\left(\mathbf{R}^{n}\right)$ satisfying supp $T \subset\{0\},(2.6),(2.7)$,

$$
\|E * T\|_{p, \mathbf{B}_{R}} \leqslant 1
$$

and $|\langle T, \bar{H}\rangle|>\gamma_{p, H}\left(\{0\}, \mathbf{B}_{R}\right) / 2$. It follows from (4.7) that $\lim _{r \downarrow 0}\|E * T\|_{p, \mathbf{B}_{r}}=0$, and hence we can find a number $\varepsilon>0$ such that $\|E * T\|_{p, \mathbf{B}_{\varepsilon}} \leqslant 1 / 4$. We conclude that

$$
\gamma_{p, H}\left(\{0\}, \mathbf{B}_{\varepsilon}\right) \geqslant|\langle 4 T, \bar{H}\rangle|>2 \gamma_{p, H}\left(\{0\}, \mathbf{B}_{R}\right) .
$$

However, it follows from Proposition 4.5 and the hypothesis $q(m-l)=n$ that $\gamma_{p, H}\left(\{0\}, \mathbf{B}_{\varepsilon}\right)=\gamma_{p, H}\left(\{0\}, \mathbf{B}_{R}\right)$, and this contradiction proves (4.2).

(b) Since $\Omega$ is bounded, we may write $\Omega \subset \mathbf{B}_{R}$ for some $R>0$. Let $H \in \mathscr{P}_{\lambda} \backslash\{0\}$. If $a \in \Omega$, then by Proposition 4.2 and the monotonicity property (4.2) we have

$$
\gamma_{p, H}(\{a\}, \Omega)=\gamma_{p, H}(\{0\}, \Omega+\{-a\}) \geqslant \gamma_{p, H}\left(\{0\}, \mathbf{B}_{2 R}\right) .
$$

To obtain a lower bound for $\gamma_{p, H}\left(\{0\}, \mathbf{B}_{2 R}\right)$, we let $T=H(D) \boldsymbol{\delta}$. If $I \in \mathscr{P}_{k}$, where $\mathbf{N} \ni k \leqslant l$, then

$$
\langle T, \bar{I}\rangle=(-1)^{l}\langle\delta, H(D) \bar{I}\rangle= \begin{cases}0 & \text { if } k<l \\ (-1)^{l} \cdot\{H, I\} & \text { if } k=l\end{cases}
$$

If $|\alpha|=l$, it follows from the hypothesis $q(m-l)>n$ that $Y_{\alpha}(D) E \in \mathbf{L}_{p}\left(\mathbf{R}^{n}\right.$, loc $)$. We may write $H=\sum_{|\alpha|=l} c_{\alpha} Y_{\alpha}$, for some choice of complex numbers $c_{\alpha}$; then $\sum_{|\alpha|=l}\left|c_{\alpha}\right| \leqslant d_{l}^{1 / 2}\{H, H\}^{l / 2}$, and hence

$$
\text { (4.10) }\|E * T\|_{p, \mathbf{B}_{2 R}}=\|H(D) E\|_{p, \mathbf{B}_{2 R}} \leqslant d_{l}^{1 / 2}\{H, H\}^{1 / 2} \sup _{|\alpha|=l}\left\|Y_{\alpha}(D) E\right\|_{p, \mathbf{B}_{2 R}} \text {. }
$$

From (4.9) and (4.10) we see that there exists a constant $C=C(l, \Omega)>0$ such that $\gamma_{p, H}\left(\{0\}, \mathbf{B}_{2 R}\right) \geqslant C\{H, H\}^{1 / 2}$, and (b) then follows from (4.8). 
5. The localization operator. If $g \in C_{0}^{\infty}\left(\mathbf{R}^{n}\right)$, we define the localization operator $\mathfrak{V}_{g}$ : $\mathscr{Q}^{\prime}\left(\mathbf{R}^{n}\right) \rightarrow \mathscr{Q}^{\prime}\left(\mathbf{R}^{n}\right)$ by

$$
\widetilde{V}_{g}(f)=E *[g L(D) f] \quad \text { if } f \in \mathscr{Q}^{\prime}\left(\mathbf{R}^{n}\right) .
$$

The operator $\mathscr{V}_{g}$ was considered by Vituškin [36] for $L(D)=\partial / \partial \bar{z}$ and $E(z)=$ $\mathrm{i} / \pi z$, and has been considered in [9 and 29] for elliptic operators $L(D)$ and their fundamental solutions $E$. We may use the operator $\mathcal{V}_{g}$ to "localize the singularities of solutions of $L(D) u=0$," in the following sense. If $g \in C_{0}^{\infty}\left(\mathbf{R}^{n}\right)$ and $f \in \mathscr{Q}^{\prime}\left(\mathbf{R}^{n}\right)$, we clearly have $L(D)\left[\mathcal{V}_{g}(f)\right]=[L(D) E] *[g L(D) f]=g L(D) f$, and hence

$$
\operatorname{supp} L(D)\left[\mathcal{T}_{g}(f)\right] \subset \operatorname{supp} g \cap \operatorname{supp} L(D) f .
$$

We therefore have $L(D)\left[\mathcal{V}_{g}(f)\right]=0$ on any open set where $g=0$, and also on any open set where $L(D) f=0$. These ideas have been used in the study of removable singularities for solutions of partial differential equations by Harvey and Polking [9, proof of Theorem 1.4], who noted an alternate formula for the localization operator $[9,(1.5)]$ which we give as Lemma 5.2. This alternate formula will then be used in proving an $\mathbf{L}_{p}$ estimate for the localization operator in Lemma 5.3, given by Lindberg [25] for $L(D)=\partial / \partial \bar{z}$ and $E(z)=1 / \pi z$.

Lemma 5.1. Let $T \in \mathcal{E}^{\prime}\left(\mathbf{R}^{n}\right)$, and let $f=E * T$. If $g \in C_{0}^{\infty}\left(\mathbf{R}^{n}\right)$ is identically equal to one on a neighborhood of $\operatorname{supp} T$, then $\mathcal{V}_{g}(f)=f$.

Proof. The hypothesis guarantees that $(1-g) T$ is the zero distribution on $\mathbf{R}^{n}$, and hence

$$
\begin{aligned}
f-\mathcal{V}_{g}(f) & =E * T-E *[g L(D)(E * T)] \\
& =E *[(1-g) T]=E * 0=0 .
\end{aligned}
$$

If $\alpha \in \mathbf{N}^{n}$, we let $L^{(\alpha)}$ denote the polynomial $D^{\alpha} L$.

Lemma 5.2. If $g \in C_{0}^{\infty}\left(\mathbf{R}^{n}\right)$ and $f \in \mathscr{D}^{\prime}\left(\mathbf{R}^{n}\right)$, then

$$
\mathcal{V}_{g}(f)=\sum_{\alpha \in \mathbf{N}^{n}} \frac{(-1)^{|\alpha|}}{\alpha !}\left(L^{(\alpha)}(D) E\right) *\left(f D^{\alpha} g\right) .
$$

PROOF. For the reader's convenience we recall here the proof from [9]. If $\phi \in C_{0}^{\infty}\left(\mathbf{R}^{n}\right)$, then Hörmander's generalization of the Leibniz formula [19, (1.4.12)] gives

$$
\begin{aligned}
\langle g L(D) f, \phi\rangle & =(-1)^{m}\langle f, L(D)(\phi g)\rangle \\
& =(-1)^{m}\left\langle f, \sum_{\alpha \in \mathbf{N}^{n}} \frac{1}{\alpha !}\left(D^{\alpha} g\right) L^{(\alpha)}(D) \phi\right\rangle \\
& =\left\langle\sum_{\alpha \in \mathbf{N}^{n}} \frac{(-1)^{|\alpha|}}{\alpha !} L^{(\alpha)}(D)\left(f D^{\alpha} g\right), \phi\right\rangle .
\end{aligned}
$$

Equating the first and last expressions yields a formula for $g L(D) f$ which we substitute in (5.1) to yield (5.3). 
Lemma 5.3. There exists a constant $C$ with the following property. If $a \in \mathbf{R}^{n}, \delta>0$, $c \in \mathbf{C}, f \in \mathbf{L}_{p}\left(\mathbf{R}^{n}\right.$, loc $)$ and $g \in C_{0}^{\infty}\left(\mathbf{B}_{\delta}(a)\right)$, then

$$
\left\|\mathscr{V}_{g}(f)\right\|_{p, \mathbf{B}_{2 \delta}(a)} \leqslant C\|f-c\|_{p, \mathbf{B}_{\delta}(a)} \sum_{|\alpha| \leqslant m} \delta^{|\alpha|}\left\|D^{\alpha} g\right\|_{\infty, \mathbf{R}^{n}}
$$

Proof. By use of Lemma 5.2 we will be able to give a proof of Lemma 5.3 which is similar to the proof of $\left[25\right.$, The $T_{\phi}$-operator, (e)].

For each multi-index $\beta \in \mathbf{N}^{n}$ satisfying $|\beta|<m$ we have $D^{\beta} E \in \mathbf{L}_{1}\left(\mathbf{R}^{n}\right.$, loc $)$, and the restriction of $D^{\beta} E$ to $\mathbf{R}^{n} \backslash\{0\}$ is continuous and homogeneous of degree $m-n-|\beta|$; hence there exists a constant $C_{\beta}$ such that

$$
\left\|D^{\beta} E\right\|_{1, \mathbf{B}_{r}} \leqslant C_{\beta} r^{m-|\beta|} \quad \text { if } r>0 .
$$

From Lemma 5.2 we see that there exist complex numbers $c_{\alpha \beta}$ for $\alpha, \beta \in \mathbf{N}^{n}$, $|\alpha| \neq 0,|\alpha+\beta|=m$, such that

$$
\mathcal{V}_{g}(f)=f g+\sum_{\substack{|\alpha| \neq 0 \\|\alpha+\beta|=m}} c_{\alpha \beta}\left(D^{\beta} E\right) *\left(f D^{\alpha} g\right) .
$$

If $B=\mathbf{B}_{\delta}(a)$, we then obtain

$$
\begin{aligned}
\left\|\mathscr{V}_{g}(f)\right\|_{p, 2 B} & \leqslant\|f g\|_{p, 2 B}+\sum_{\substack{|\alpha| \neq 0 \\
|\alpha+\beta|=m}}\left|c_{\alpha \beta}\right|\left\|\left(D^{\beta} E\right) *\left(f D^{\alpha} g\right)\right\|_{p, 2 B} . \\
& \leqslant\|f\|_{p, B}\|g\|_{\infty, B}+\sum_{\substack{|\alpha| \neq 0 \\
|\alpha+\beta|=m}}\left|c_{\alpha \beta}\right|\left\|D^{\beta} E\right\|_{1, \mathbf{B}_{3 \delta}(0)}\left\|f D^{\alpha} g\right\|_{p, B} \\
& \leqslant\|f\|_{p, B}\|g\|_{\infty, B}+\sum_{\substack{|\alpha| \neq 0 \\
|\alpha+\beta|=m}}\left|c_{\alpha \beta}\right| C_{\beta}(3 \delta)^{m-|\beta|}\|f\|_{p, B}\left\|D^{\alpha} g\right\|_{\infty, B},
\end{aligned}
$$

where the three inequalities follow, respectively, from (5.5), Young's inequality and (5.4). This proves the lemma in the special case $c=0$. If $c \in \mathbf{C}$ is arbitrary, the lemma follows by applying this special case to the function $f-c \in \mathbf{L}_{p}\left(\mathbf{R}^{n}\right.$, loc $)$.

6. Proof of necessary conditions for approximation. In this section we will prove the implication (a) $\Rightarrow(b)$ in Theorem 2.1. We suppose that condition (a) of that theorem holds. Let $\mathbf{N} \ni l \leqslant m-1$ and $H \in \mathcal{P}_{\backslash} \backslash\{0\}$, and let $G$ and $\Omega$ be open subsets of $\mathbf{R}^{n}$ satisfying $G \Subset \Omega \Subset \mathbf{R}^{n}$. In view of the monotonicity property (4.1) it suffices to prove that

$$
\gamma_{p, H}(G \backslash X, \Omega) \geqslant \gamma_{p, H}(G \backslash \text { int } X, \Omega) .
$$

We will assume that

$$
\gamma_{p, H}(G \backslash \text { int } X, \Omega)>0,
$$

for otherwise (6.1) is obvious. From (6.2) it follows that

$$
G \backslash X \neq \phi ;
$$

in fact, if (6.3) fails, we obtain $G \subset X$ and hence $G \subset$ int $X$, so (6.2) fails. 
In view of (6.3) there exists a function $\psi \in C_{0}^{\infty}(G \backslash X)$ satisfying $\int \psi d \lambda \neq 0$; we let this function $\psi$ be fixed for the rest of this section. For each $k \in \mathbf{N}$ we let $\left\{\alpha \in \mathbf{N}^{n}:|\alpha|=k\right\}$ carry a fixed ordering, so that each point of $\mathbf{C}^{d_{k}}$ may be written as $\left\{c_{\alpha}\right\}_{|\alpha|=k}$ for some choice of complex numbers $c_{\alpha}$, and we make the following three observations.

$1^{\circ}$. The mapping

$$
\mathscr{P}_{k} \ni J \rightarrow\left\{\left\langle J(D) \psi, Y_{\alpha}\right\rangle\right\}_{|\alpha|=k} \in \mathbf{C}^{d_{k}}
$$

is an isomorphism of complex vector spaces.

If $J \in \mathscr{P}_{k}$ and $|\alpha|=k$, then

$$
\left\langle J(D) \psi, Y_{\alpha}\right\rangle=(-1)^{k}\left\langle\psi, J(D) Y_{\alpha}\right\rangle=(-1)^{k}\left\{J, Y_{\alpha}\right\} \int \psi d \lambda,
$$

so $1^{\circ}$ is obvious.

$2^{\circ}$. For each $J \in \mathscr{P}_{k}$ we have $\langle J(D) \psi, \bar{I}\rangle=0$ if $I \in \mathscr{P}_{0} \cup \cdots \cup \mathscr{P}_{k-1}$.

If $J \in \mathscr{P}_{k}$ and $I$ is any polynomial, we have $\langle J(D) \psi, \bar{I}\rangle=(-1)^{k}\langle\psi, J(D) \bar{I}\rangle$, from which $2^{\circ}$ follows.

$3^{\circ}$. The mapping

$$
\mathscr{P}_{k} \ni J \rightarrow\|E * J(D) \psi\|_{p, \Omega}
$$

is a norm on $\mathscr{P}_{k}$.

If $J \in \mathscr{P}_{k}$, then $E * J(D) \psi \in C^{\infty}\left(\mathbf{R}^{n}\right)$, and hence $\|E * J(D) \psi\|_{p, \Omega}<\infty$. If $J \in \mathscr{P}_{k}$ and $\|E * J(D) \psi\|_{p, \Omega}=0$, it follows from Lemma 3.1 that $J(D) \psi=0$ in $\varepsilon^{\prime}\left(\mathbf{R}^{n}\right)$; since (6.4) is injective, it follows that $J=0$. The other axioms for a norm are clearly satisfied, so $3^{\circ}$ holds.

The following notation will be used for the rest of this section: for each $k \in \mathbf{N}, \tau_{k}$ denotes the norm of the inverse of the mapping (6.4) when $\mathscr{P}_{k}$ carries the norm (6.5) and $\mathbf{C}^{d_{k}}$ carries the norm $\left\|\left\{c_{\alpha}\right\}\right\|=\sup \left\{\left|c_{\alpha}\right|:|\alpha|=k\right\}$.

We now let $T \in \mathcal{E}^{\prime}\left(\mathbf{R}^{n}\right)$ be a fixed distribution satisfying

$$
\begin{gathered}
\operatorname{supp} T \subset G \backslash \text { int } X \subset G, \\
\langle T, \bar{I}\rangle=0 \text { if } I \in \mathscr{P}_{0} \cup \cdots \cup \mathscr{P}_{l-1}, \\
\langle T, \bar{I}\rangle=0 \text { if } I \in \mathscr{P}_{l} \text { and }\{H, I\}=0, \\
E * T \in \mathbf{L}_{p}\left(\mathbf{R}^{n}, \text { loc }\right) \text { and } 0<\|E * T\|_{p, \Omega} \leqslant 1 .
\end{gathered}
$$

Our goal is to prove that

$$
\gamma_{p, H}(G \backslash X, \Omega) \geqslant|\langle T, \bar{H}\rangle| /\|E * T\|_{p, \Omega} .
$$

In view of (6.2) and Remark 3 in $\S 4$, this will imply (6.1), as desired.

We let $f=E * T$, and we let $g \in C_{0}^{\infty}(G)$ be a fixed function which is identically equal to one on a neighborhood of $\operatorname{supp} T$. It follows from Lemma 5.1 that $\mathcal{V}_{g}(f)=f$.

Now select $R>0$ large enough that $X \cup \bar{\Omega} \subset \mathbf{B}_{R}$, and note that (6.6) implies that $L(D) f=0$ on int $X$. From condition (a) of Theorem 2.1 we conclude that for each $j \in \mathbf{N}$ there exists an open set $A_{j}$ satisfying $X \subset A_{j} \subset \mathbf{B}_{R}$, and a function $u_{j} \in \mathbf{L}_{p}\left(A_{j}\right)$, such that $L(D) u_{j}=0$ on $A_{j}$ and $\left\|u_{j}-f\right\|_{p, X}<1 / 3 j$; replacing $A_{j}$ by a smaller 
neighborhood of $X$ (if necessary) we may also assume that $\left\|u_{j}\right\|_{p, A_{j} \backslash X}<1 / 3 j$ and $\|f\|_{p, A_{\supset} \backslash X}<1 / 3 j$; hence $\left\|u_{j}-f\right\|_{p, A_{j}}<1 / j$. For each $j \in \mathbf{N}$ we define $u_{j}$ to be identically equal to $f$ on $\mathbf{R}^{n} \backslash A_{j}$; we note that $u_{j} \in \mathbf{L}_{p}\left(\mathbf{R}^{n}\right.$, loc $)$ and that $\operatorname{supp}\left[g L(D) u_{j}\right]$ is a compact subset of $G \backslash X$. If $j \in \mathbf{N}$ we define $v_{j}=\mathcal{V}_{g}\left(u_{j}\right)=$ $E *\left[g L(D) u_{j}\right]$; we conclude from Lemma 5.3 that $v_{j} \in \mathbf{L}_{p}\left(\mathbf{R}^{n}, \operatorname{loc}\right)$ and that

$$
\begin{aligned}
\left\|v_{j}-f\right\|_{p, \Omega} & =\left\|\mho_{g}\left(u_{j}-f\right)\right\|_{p, \Omega} \leqslant\left\|\mho_{g}\left(u_{j}-f\right)\right\|_{p, \mathbf{B}_{R}} \\
& \leqslant C_{1}\left\|u_{j}-f\right\|_{p, \mathbf{B}_{R}}=C_{1}\left\|u_{j}-f\right\|_{p, A_{j}} \leqslant C_{1} j^{-1}
\end{aligned}
$$

here $C_{1}=C_{2} \Sigma_{|\alpha| \leqslant m} R^{|\alpha|}\left\|D^{\alpha} g\right\|_{\infty, \mathbf{R}^{n}}$, where $C_{2}$ is the constant $C$ of Lemma 5.3. We conclude from Corollary 3.3 that, for $j \in \mathbf{N}$, we have

$$
\left|\left\langle g L(D) u_{j}-T, \bar{I}\right\rangle\right| \leqslant C_{1} C_{3}\{I, I\}^{1 / 2} j^{-1} \quad \text { if } I \in \mathscr{P}_{0} \cup \cdots \cup \mathscr{P}_{l},
$$

where $C_{3}$ denotes the constant $C(l, \bar{G}, \Omega)$ of Corollary 3.3.

We now claim that for each $j \in \mathbf{N}$ there exists a function $W_{j} \in C_{0}^{\infty}(G \backslash X) \subset$ $\mathcal{E}^{\prime}\left(\mathbf{R}^{n}\right)$ such that

$$
\left\langle W_{j}, \bar{I}\right\rangle=\left\langle g L(D) u_{j}-T, \bar{I}\right\rangle \quad \text { if } I \in \mathscr{P}_{0} \cup \cdots \cup \mathscr{P}_{l},
$$

and

$$
\left\|E * W_{j}\right\|_{p, \Omega} \leqslant \zeta j^{-1}
$$

where $\zeta$ is a constant depending on $l, G, \Omega, R, g$ and on $\tau_{0}, \tau_{1}, \ldots, \tau_{l}$. Assuming for the moment that this claim has been proved, and writing $w_{j}=E * W_{j}$ for each $j \in \mathbf{N}$, we conclude from (6.9), (6.11) and (6.14) that, for sufficiently large $j$, we have $\left\|w_{j}-v_{j}\right\|_{p, \Omega}>0$, and hence from (6.7), (6.8) and (6.13) we obtain

$$
\begin{aligned}
\gamma_{p, H}(G \backslash X, \Omega) & \geqslant\left|\left\langle g L(D) u_{j}-W_{j}, \bar{H}\right\rangle\right| /\left\|w_{j}-v_{j}\right\|_{p, \Omega} \\
& =|\langle T, \bar{H}\rangle| /\left\|w_{j}-v_{j}\right\|_{p, \Omega} .
\end{aligned}
$$

Letting $j \rightarrow \infty$ and applying (6.11) and (6.14), we obtain

$$
\gamma_{p, H}(G \backslash X, \Omega) \geqslant|\langle T, \bar{H}\rangle| /\|f\|_{p, \Omega},
$$

which is the desired inequality (6.10).

We now prove the claim of the preceding paragraph. To do this, we use induction on $\nu$ to prove the following assertion for $\nu \in\{0,1, \ldots, l\}$.

$\Pi_{\nu}:$ For each $j \in \mathbf{N}$ there exists a function $W_{j}^{(\nu)} \in C_{0}^{\infty}(G \backslash X) \subset \mathcal{E}^{\prime}\left(\mathbf{R}^{n}\right)$ such that

$$
\left\langle W_{j}^{(\nu)}, \bar{I}\right\rangle=\left\langle g L(D) u_{j}-T, \bar{I}\right\rangle \quad \text { if } I \in \mathscr{P}_{0} \cup \cdots \cup \mathscr{P}_{\nu},
$$

and $\left\|E * W_{j}^{(\nu)}\right\|_{p, \Omega} \leqslant \zeta_{\nu} j^{-1}$.

Here the constants $\zeta_{\nu}$ are defined inductively by the equations $\zeta_{0}=\tau_{0} C_{1} C_{3}$ and $\zeta_{\nu}=\tau_{\nu} C_{1} C_{3}+\left(1+\tau_{\nu} C_{3}\right) \zeta_{\nu-1}$ if $1 \leqslant \nu \leqslant l$. Since $\Pi_{l}$ implies the claim, with $\zeta=\zeta_{l}$, and $W_{j}=W_{j}^{(l)}$ for each $j \in \mathbf{N}$, this will complete the proof of (6.10).

To prove $\Pi_{0}$, we apply $1^{\circ}$ and $3^{\circ}$ (for $k=0$ ) and the definition of $\tau_{0}$. We conclude that, for each $j \in \mathbf{N}$, there exists a polynomial $J_{j}^{(0)} \in \mathscr{P}_{0}$ such that the 
function $W_{j}^{(0)}=J_{j}^{(0)}(D) \psi \in C_{0}^{\infty}(G \backslash X) \subset \mathcal{E}^{\prime}\left(\mathbf{R}^{n}\right)$ satisfies $\left\langle W_{j}^{(0)}, 1\right\rangle=\left\langle g L(D) u_{j}-\right.$ $T, 1>$ and

$$
\left\|E * W_{j}^{(0)}\right\|_{p, \Omega} \leqslant \tau_{0}\left|\left\langle g L(D) u_{j}-T, 1\right\rangle\right| \leqslant C_{1} C_{3} \tau_{0} j^{-1},
$$

where the last inequality follows from (6.12). This proves $\Pi_{0}$.

Now let $\nu \in\{1, \ldots, l\}$ be fixed, and suppose $\Pi_{\nu-1}$ has been proved. We let $j \in \mathbf{N}$ be fixed. If $W_{j}^{(\nu-1)} \in C_{0}^{\infty}(G \backslash X)$ is the function given by $\Pi_{\nu-1}$, we define $S=$ $g L(D) u_{j}-T-W_{j}^{(\nu-1)}$. Applying $1^{\circ}, 2^{\circ}$ and $3^{\circ}$ (for $k=\nu$ ) and the definition of $\tau_{\nu}$, we see that there exists a polynomial $J \in \mathscr{P}_{\nu}$ such that the function $W^{\prime}=J(D) \psi \in$ $C_{0}^{\infty}(G \backslash X) \subset \mathcal{E}^{\prime}\left(\mathbf{R}^{n}\right)$ satisfies

$$
\begin{gathered}
\left\langle W^{\prime}, Y_{\alpha}\right\rangle=\left\langle S, Y_{\alpha}\right\rangle \quad \text { if }|\alpha|=\nu, \\
\left\langle W^{\prime}, \bar{I}\right\rangle=0 \quad \text { if } I \in \mathscr{P}_{0} \cup \cdots \cup \mathscr{P}_{\nu-1},
\end{gathered}
$$

and

$$
\left\|E * W^{\prime}\right\|_{p, \Omega} \leqslant \tau_{\nu} \sup _{|\alpha|=\nu}\left|\left\langle S, Y_{\alpha}\right\rangle\right|
$$

We then obtain

$$
\begin{aligned}
\left\|E * W^{\prime}\right\|_{p, \Omega} & \leqslant \tau_{\nu}\left[\sup _{|\alpha|=\nu}\left|\left\langle g L(D) u_{j}-T, Y_{\alpha}\right\rangle\right|+\sup _{|\alpha|=\nu}\left|\left\langle W_{j}^{(\nu-1)}, Y_{\alpha}\right\rangle\right|\right] \\
& \leqslant \tau_{\nu}\left[C_{1} C_{3} j^{-1}+C_{3}\left\|E * W_{j}^{(\nu-1)}\right\|_{p, \Omega}\right] \\
& \leqslant \tau_{\nu} C_{3}\left(C_{1}+\zeta_{\nu-1}\right) j^{-1}
\end{aligned}
$$

where the first inequality follows from (6.15), the second inequality from (6.12) and Corollary 3.3, and the third inequality from the induction hypothesis. Thus we see that $\Pi_{\nu}$ holds with $W_{j}^{(\nu)}=W_{j}^{(\nu-1)}+W^{\prime}$ for each $j \in \mathbf{N}$.

7. Proof of sufficient conditions for approximation. In this section we will complete the proof of Theorem 2.1 ; since the implication $(b) \Rightarrow(c)$ is obvious, it remains to prove the implication (c) $\Rightarrow$ (a). Our techniques extend those used by Lindberg [24, 25] for $L(D)=\partial / \partial \bar{z}$, which are based on those used by Vituškin [36]. Lemma 7.1 was given in [25, Lemma] for $L(D)=\partial / \partial \bar{z}$ and $E(z)=1 / \pi z$; Lemmas 7.2 and 7.3 summarize well-known facts associated with cubical decompositions of $\mathbf{R}^{n}$, and Lemma 7.4 is essentially given in $[25, \mathrm{p} .66]$. We denote by $\omega$ the $(n-1)$-dimensional area of the unit sphere in $\mathbf{R}^{n}$, so that $\omega=n \lambda\left(\mathbf{B}_{1}\right)$. We use $\Sigma_{j}$ as a shortened notation for $\sum_{j=0}^{\infty}$.

LEMMA 7.1. For each positive integer $Z$, there exists a constant $C=C(Z)$ with the following property. Let $\delta>0$, and let $\left\{B_{j}\right\}_{j \in \mathbf{N}}$ be a sequence of open balls of radius $\delta$ in $\mathbf{R}^{n}$ such that no point of $\mathbf{R}^{n}$ lies in more than $Z$ of these balls. Let $\left\{T_{j}\right\}_{j \in \mathbf{N}}$ be a sequence in $\mathcal{E}^{\prime}\left(\mathbf{R}^{n}\right)$ such that, for each $j \in \mathbf{N}$, we have

$$
\begin{gathered}
\operatorname{supp} T_{j} \subset \bar{B}_{j}, \\
\left\langle T_{j}, I\right\rangle=0 \quad \text { if } I \in \mathscr{P}_{0} \cup \cdots \cup \mathscr{P}_{m-1},
\end{gathered}
$$


and

$$
f_{j} \equiv E * T_{j} \in \mathbf{L}_{p}\left(\mathbf{R}^{n}, \text { loc }\right) .
$$

If $\mathcal{G}$ is a finite subset of $\mathbf{N}$, then

$$
\left\|\sum_{j \in \mathcal{g}} f_{j}\right\|_{p, \mathbf{R}^{n}}^{p} \leqslant C \sum_{j}\left\|f_{j}\right\|_{p, 2 B_{j}}^{p} .
$$

Proof. The form of the proof will be similar to that of [25, Lemma].

By considering the mapping $\mathbf{R}^{n} \ni x \rightarrow x / \delta \in \mathbf{R}^{n}$, and applying (4.4), (4.5) and Lemmas 4.3 and 4.4 , we may reduce the proof to the case $\delta=1$. We therefore assume that $\delta=1$ throughout the proof, and we write $B_{j}=\mathbf{B}_{1}\left(x_{j}\right)$.

We first prove Lemma 7.1 under the additional hypothesis that for each $j \in \mathbf{N}$ we have

$$
\left\langle T_{j}, I\right\rangle=0 \quad \text { if } I \in \mathscr{P}_{m} .
$$

If $a \in \mathbf{R}^{n}$ and $k \in\{1,2, \ldots\}$, we consider the set

$$
\mathbb{Q}_{k}(a)=\left\{x \in \mathbf{R}^{n}: 2 k-2 \leqslant|x-a|<2 k\right\},
$$

we let $N_{k}(a)$ denote the number of the centers $x_{j}$ which lie in $\mathcal{Q}_{k}(a)$, and we make the following three observations.

$1^{\circ} . N_{k}(a) \leqslant C_{1} k^{n-1}$, where $C_{1}=n 4^{n} Z$.

To prove $1^{\circ}$, we note that if $k \geqslant 2$, the $N_{k}(a)$ balls $B_{j}$ having centers $x_{j} \in \mathbb{Q}_{k}(a)$ must all lie in the set $\left\{x \in \mathbf{R}^{n}: 2 k-3 \leqslant|x-a| \leqslant 2 k+1\right\}$; since each point of $\mathbf{R}^{n}$ lies in at most $Z$ of the balls $B_{j}$, we conclude that

$$
\begin{aligned}
N_{k}(a) \lambda\left(\mathbf{B}_{1}\right) & \leqslant Z \lambda\left(\left\{x \in \mathbf{R}^{n}: 2 k-3 \leqslant|x-a| \leqslant 2 k+1\right\}\right) \\
& =Z \omega \int_{2 k-3}^{2 k+1} r^{n-1} d r \\
& \leqslant 4 n Z \lambda\left(\mathbf{B}_{1}\right)(2 k+1)^{n-1} \\
& \leqslant n 4^{n} Z \lambda\left(\mathbf{B}_{1}\right) k^{n-1},
\end{aligned}
$$

as desired. For $k=1$ a similar argument yields the desired result.

$2^{\circ} .\left(\sum_{x_{j} \in \mathbb{Q}_{k}(a)}\left|f_{j}(a)\right|\right)^{p} \leqslant\left(C_{1} k^{n-1}\right)^{p / q} \sum_{x_{j} \in \mathbb{Q}_{k}(a)}\left|f_{j}(a)\right|^{p}$.

This follows from $1^{\circ}$ and (3.2).

$3^{\circ}$. $\int_{\mathbb{Q}_{k}\left(x_{j}\right)}\left|f_{j}\right|^{p} d \lambda \leqslant C_{2}\left\|f_{j}\right\|_{p, \mathbf{B}_{2}\left(x_{j}\right)}^{p} k^{-n(p-1)-p-1}$; here $C_{2}=\max \left\{1,2 C_{3}^{p} \omega\right\}$, where $C_{3}$ denotes the constant $C(m+1)$ of Lemma 3.7.

For $k=1,3^{\circ}$ is obvious. Thus we may prove $3^{\circ}$ under the assumption that $k \geqslant 2$. We then have $\mathscr{Q}_{k}\left(x_{j}\right) \subset\left\{x \in \mathbf{R}^{n}:\left|x-x_{j}\right| \geqslant 2\right\}$, and hence we obtain from Lemma 3.7

$$
\begin{aligned}
\int_{\mathbb{Q}_{k}\left(x_{j}\right)}\left|f_{j}\right|^{p} d \lambda & \leqslant C_{3}^{p} \omega\left\|f_{j}\right\|_{p, \mathbf{B}_{2}\left(x_{j}\right)}^{p} \int_{2 k-2}^{2 k} r^{-n(p-1)-p-1} d r \\
& \leqslant 2 C_{3}^{p} \omega\left\|f_{j}\right\|_{p, \mathbf{B}_{2}\left(x_{j}\right)}^{p}(2 k-2)^{-n(p-1)-p-1} \\
& \leqslant C_{2}\left\|f_{j}\right\|_{p, \mathbf{B}_{2}\left(x_{j}\right)}^{p} k^{-n(p-1)-p-1}
\end{aligned}
$$

which gives $3^{\circ}$. 
We now obtain

$$
\begin{aligned}
\left\|\sum_{j \in \mathcal{G}} f_{j}\right\|_{p, \mathbf{R}^{n}} & \leqslant\left[\int\left(\sum_{j}\left|f_{j}(x)\right|\right)^{p} d \lambda(x)\right]^{1 / p}=\left[\int\left(\sum_{k=1}^{\infty} \sum_{x_{j} \in \mathbb{Q}_{k}(x)}\left|f_{j}(x)\right|\right)^{p} d \lambda(x)\right]^{1 / p} \\
& \leqslant \sum_{k=1}^{\infty}\left[\int\left(\sum_{x_{j} \in \mathbb{Q}_{k}(x)}\left|f_{j}(x)\right|\right)^{p} d \lambda(x)\right]^{1 / p} \\
& \leqslant \sum_{k=1}^{\infty}\left[\int\left(C_{1} k^{n-1}\right)^{p / q} \sum_{x_{j} \in \mathbb{Q}_{k}(x)}\left|f_{j}(x)\right|^{p} d \lambda(x)\right]^{1 / p} \\
& =\sum_{k=1}^{\infty}\left(C_{1} k^{n-1}\right)^{1 / q}\left[\sum_{j} \int_{\mathbb{Q}_{k}\left(x_{j}\right)}\left|f_{j}(x)\right|^{p} d \lambda(x)\right]^{i / p} \\
& \leqslant C_{1}^{1 / q} C_{2}^{1 / p} \sum_{k=1}^{\infty} k^{-2}\left[\sum_{j}\left\|f_{j}\right\|_{p, \mathbf{B}_{2}\left(x_{j}\right)}^{p}\right]^{1 / p} \equiv C_{4}\left[\sum_{j}\left\|f_{j}\right\|_{p, \mathbf{B}_{2}\left(x_{j}\right)}^{p}\right]^{1 / p}
\end{aligned}
$$

where the second, third and final inequalities follow, respectively, from Minkowski's inequality, $2^{\circ}$ and $3^{\circ}$; and the symbol $\equiv$ in the last line indicates that we define $C_{4}=C_{1}^{1 / q} C_{2}^{1 / p} \sum_{k=1}^{\infty} k^{-2}$. This proves Lemma 7.1, with $C=C_{4}^{p}$, under the additional hypothesis (7.5).

We now prove Lemma 7.1 in the general case. Let the distributions $T_{j}$ and functions $f_{j} \equiv E * T_{j}$ satisfy the hypotheses of the lemma. For each $j \in \mathbf{N}$ we define

$$
U_{j}=\frac{(-1)^{m}}{\lambda\left(\mathbf{B}_{1}\right)} \sum_{|\alpha|=m}\left\langle T_{j}, Y_{\alpha}\right\rangle Y_{\alpha}(D) \chi_{B_{j}} \in \mathcal{E}^{\prime}\left(\mathbf{R}^{n}\right)
$$

and $u_{j}=E * U_{j}$; it follows from Lemma 3.4 that $u_{j} \in \mathbf{L}_{p}\left(\mathbf{R}^{n}\right)$. Moreover, if $j \in \mathbf{N}$, $|\alpha|=m$ and $I \in \mathcal{P}_{k}$, where $\mathbf{N} \ni k \leqslant m$, then

$$
\begin{aligned}
\left\langle Y_{\alpha}(D) \chi_{B_{j}}, I\right\rangle & =(-1)^{m}\left\langle\chi_{B_{j}}, Y_{\alpha}(D) I\right\rangle \\
& = \begin{cases}0 & \text { if } k<m, \\
(-1)^{m} \lambda\left(\mathbf{B}_{1}\right)\left\{I, Y_{\alpha}\right\} & \text { if } k=m .\end{cases}
\end{aligned}
$$

It follows that the sequence $\left\{T_{j}-U_{j}\right\}_{j \in \mathbf{N}}$ in $\mathcal{E}^{\prime}\left(\mathbf{R}^{n}\right)$ satisfies the hypotheses of the first part of the proof, and hence

$$
\left\|\sum_{j \in \mathcal{G}}\left(f_{j}-u_{j}\right)\right\|_{p, \mathbf{R}^{n}}^{p} \leqslant C_{4}^{p} \sum_{j}\left\|f_{j}-u_{j}\right\|_{p, \mathbf{B}_{2}\left(x_{j}\right)}^{p} .
$$

To finish the proof of Lemma 7.1, we need further estimates involving the functions $u_{j}$. We first note that if $j \in \mathbf{N}$, and $\left(f_{j}\right)_{-x_{j}}$ is the translated function $f_{j}\left(\cdot+x_{j}\right) \in \mathbf{L}_{p}\left(\mathbf{R}^{n}\right.$, loc $)$, then the distribution $L(D)\left[\left(f_{j}\right)_{-x_{j}}\right]$ has support in $\overline{\mathbf{B}_{1}}$, and hence from Lemma 4.1 and Corollary 3.3 we obtain

$$
\begin{aligned}
\left|\left\langle T_{j}, Y_{\alpha}\right\rangle\right| & =\left|\left\langle L(D)\left[\left(f_{j}\right)_{-x_{j}}\right], Y_{\alpha}\right\rangle\right| \leqslant C_{5}\left\|\left(f_{j}\right)_{-x_{j}}\right\|_{p, \mathbf{B}_{2}} \\
& =C_{5}\left\|f_{j}\right\|_{p, \mathbf{B}_{2}\left(x_{j}\right)}, \quad \text { if }|\alpha|=m,
\end{aligned}
$$


where $C_{5}$ denotes the constant $C\left(m, \overline{\mathbf{B}_{1}}, \mathbf{B}_{2}\right)$ of Corollary 3.3; we conclude that

$$
\begin{aligned}
\left\|u_{j}\right\|_{p, \mathbf{R}^{n}} & \leqslant C_{5} C_{6} d_{m} \lambda\left(\mathbf{B}_{1}\right)^{-1}\left\|f_{j}\right\|_{p, \mathbf{B}_{2}\left(x_{j}\right)}\left\|\chi_{B_{j}}\right\|_{p, \mathbf{R}^{n}} \\
& =C_{5} C_{6} d_{m} \lambda\left(\mathbf{B}_{1}\right)^{-1 / q}\left\|f_{j}\right\|_{p, \mathbf{B}_{2}\left(x_{j}\right)} \\
& \equiv C_{7}\left\|f_{j}\right\|_{p, \mathbf{B}_{2}\left(x_{j}\right)},
\end{aligned}
$$

where $C_{6}$ denotes the constant $C$ of Lemma 3.4. Moreover, we have

$$
\begin{aligned}
\int_{\mathbf{R}^{n}}\left|\sum_{j \in \mathcal{G}} u_{j}\right|^{p} d \lambda & \leqslant d_{m}^{p / q} \lambda\left(\mathbf{B}_{1}\right)^{-p} \sum_{|\alpha|=m}\left\|Y_{\alpha}(D) E *\left[\sum_{j \in \mathcal{G}}\left\langle T_{j}, Y_{\alpha}\right\rangle \chi_{B_{j}}\right]\right\|_{p, \mathbf{R}^{n}}^{p} \\
& \leqslant d_{m}^{p / q} \lambda\left(\mathbf{B}_{1}\right)^{-p} \sum_{|\alpha|=m} C_{6}^{p} \int_{\mathbf{R}^{n}}\left|\sum_{j \in \mathcal{g}}\left\langle T_{j}, Y_{\alpha}\right\rangle \chi_{B_{j}}\right|^{p} d \lambda \\
& \leqslant d_{m}^{p / q} \lambda\left(\mathbf{B}_{1}\right)^{-p} \sum_{|\alpha|=m} C_{6}^{p} \int_{\mathbf{R}^{n}} Z^{p / q} \sum_{j}\left|\left\langle T_{j}, Y_{\alpha}\right\rangle \chi_{B_{j}}\right|^{p} d \lambda \\
& \leqslant d_{m}^{p} \lambda\left(\mathbf{B}_{1}\right)^{-p / q} C_{\xi}^{p} C_{6}^{p} Z^{p / q} \sum_{j}\left\|f_{j}\right\|_{p, \mathbf{B}_{2}\left(x_{j}\right)}^{p} \\
& \equiv C_{8} \sum_{j}\left\|f_{j}\right\|_{p, \mathbf{B}_{2}\left(x_{j}\right)}^{p},
\end{aligned}
$$

where the four inequalities follow, respectively, from (3.2), Lemma 3.4, (3.2) and (7.7).

Finally, we conclude that

$$
\begin{aligned}
\int_{\mathbf{R}^{n}}\left|\sum_{j \in \mathcal{G}} f_{j}\right|^{p} d \lambda & \leqslant 2^{p / q} \int_{\mathbf{R}^{n}}\left|\sum_{j \in \mathcal{G}}\left(f_{j}-u_{j}\right)\right|^{p} d \lambda+22^{p / q} \int_{\mathbf{R}^{n}}\left|\sum_{j \in \mathcal{G}} u_{j}\right|^{p} d \lambda \\
& \leqslant 2^{p / q} C_{4}^{p} \sum_{j} \int_{\mathbf{B}_{2}\left(x_{j}\right)}\left|f_{j}-u_{j}\right|^{p} d \lambda+2^{p / q} C_{8} \sum_{j}\left\|f_{j}\right\|_{p, \mathbf{B}_{2}\left(x_{j}\right)}^{p} \\
& \leqslant 2^{2 p / q} C_{4}^{p} \sum_{j} \int_{\mathbf{B}_{2}\left(x_{j}\right)}\left(\left|f_{j}\right|^{p}+\left|u_{j}\right|^{p}\right) d \lambda+2^{p / q} C_{8} \sum_{j}\left\|f_{j}\right\|_{p, \mathbf{B}_{2}\left(x_{j}\right)}^{p} \\
& \leqslant\left(2^{2 p / q} C_{4}^{p}\left(1+C_{7}^{p}\right)+2^{p / q} C_{8}\right) \underset{j}{\sum_{j}\left\|f_{j}\right\|_{p, \mathbf{B}_{2}\left(x_{j}\right)}^{p},}
\end{aligned}
$$

where the first inequality follows from (3.2), the second inequality from (7.6) and (7.9), the third inequality from (3.2), and the last inequality from (7.8). This proves Lemma 7.1 .

For the rest of the paper we let $h \in C_{0}^{\infty}\left(\mathbf{B}_{1 / 3}\right)$ be a nonnegative function satisfying $\int h d \lambda=1$, and for each $\delta>0$ we define $h_{\delta} \in C_{0}^{\infty}\left(\mathbf{B}_{\delta / 3}\right)$ by $h_{\delta}(x)=$ $\delta^{-n} h(x / \delta)$. For each $k \in \mathrm{N}$ there exists a positive constant $M_{k}$ such that $\left|D^{\alpha} h\right| \leqslant M_{k}$ if $|\alpha|=k$. It follows from the chain rule that

$$
\left|D^{\alpha} h_{\delta}\right| \leqslant \delta^{-n-k} M_{k} \quad \text { if }|\alpha|=k .
$$


We say that a point of $\mathbf{R}^{n}$ is a lattice point if each of its $n$ coordinates is an integer, and we let $Z=Z(n)$ be the largest number of lattice points which can occur in a closed ball of radius $2 \sqrt{n}$ in $\mathbf{R}^{n}$.

We now use decompositions of $\mathbf{R}^{n}$ which are analogous to those used by Vituškin [36], Lindberg [24, 25] and others. To define these we let $\left\{a_{j}\right\}_{j \in \mathbf{N}}$ be an enumeration of all lattice points in $\mathbf{R}^{n}$. We let $\delta>0$ be fixed, and set $\delta_{0}=\delta / 2 \sqrt{n}$. We define $x_{j, \delta}=\delta_{0} a_{j} \in \mathbf{R}^{n}$ for each $j \in \mathbf{N}$. If

$$
2^{(\delta)}=\left[-\frac{\delta_{0}}{2}, \frac{\delta_{0}}{2}\right) \times \cdots \times\left[-\frac{\delta_{0}}{2}, \frac{\delta_{0}}{2}\right) \quad(n \text { times }),
$$

then $\overline{\mathcal{Q}^{(\delta)}} \subset \mathbf{B}_{\delta / 3}$. For $j \in \mathbf{N}$, the sets $\mathscr{Q}_{j, \delta}=\mathcal{Q}^{(\delta)}+x_{j, \delta}$ are disjoint, their union is $\mathbf{R}^{n}$, and $\overline{\mathcal{Q}_{j, \delta}} \subset \mathbf{B}_{\delta / 3}\left(x_{j, \delta}\right)$. We now define, for each $j \in \mathbf{N}$,

$$
g_{j, \delta}=h_{\delta} * \chi_{Q_{j, \delta}} \in C_{0}^{\infty}\left(\mathbf{R}^{n}\right) \text { and } B_{j, \delta}=\mathbf{B}_{\delta}\left(x_{j, \delta}\right) .
$$

LEMMA 7.2. Let $\delta>0$. Then:

(a) $\operatorname{supp} g_{j, \delta} \subset B_{j, \delta}$ if $j \in \mathbf{N}$,

(b) $\left|D^{\alpha} g_{j, \delta}\right| \leqslant M_{|\alpha|} 2^{-n} n^{-n / 2} \delta^{-|\alpha|}$ if $\alpha \in \mathbf{N}^{n}, j \in \mathbf{N}$, and

(c) each point $x \in \mathbf{R}^{n}$ satisfies $x \in B_{j, \delta}$ for at most $Z$ indices $j \in \mathbf{N}$.

Proof. (a) For $j \in \mathbf{N}$ we have

$$
\operatorname{supp} g_{j, \delta} \subset \operatorname{supp} h_{\delta}+\operatorname{supp} \chi_{\beth_{, \delta \delta}} \subset \mathbf{B}_{\delta / 3}+\mathbf{B}_{\delta / 3}\left(x_{j, \delta}\right) \subset B_{j, \delta} .
$$

(b) For $\alpha \in \mathbf{N}^{n}$ and $j \in \mathbf{N}$ we obtain, from (7.10),

$$
\begin{aligned}
\left|D^{\alpha} g_{j, \delta}\right| & =\left|\left(D^{\alpha} h_{\delta}\right) * \chi_{2_{j, \delta}}\right| \leqslant M_{|\alpha|} \delta^{-n-|\alpha|} \lambda\left(2_{j, \delta}\right) \\
& \leqslant M_{|\alpha|} 2^{-n} n^{-n / 2} \delta^{-|\alpha|} .
\end{aligned}
$$

(c) is obvious.

LEMMA 7.3. Let $\delta>0$, let $K \subset \mathbf{R}^{n}$ be compact, and let $\mathcal{g}_{\delta}$ be the set of all $j \in \mathbf{N}$ such that $B_{j, \delta}$ intersects $K$. Then $\sum_{j \in g_{\delta}} g_{j, \delta}(x)=1$ if $\operatorname{dist}(x, K)<\delta / 3$.

Proof. We first show that

$$
\left\{y \in \mathbf{R}^{n}: \operatorname{dist}(y, K)<2 \delta / 3\right\} \subset \bigcup_{j \in \mathcal{G}_{\delta}} \mathcal{Q}_{j, \delta} .
$$

To prove this, let $y \in \mathbf{R}^{n}$ satisfy $\operatorname{dist}(y, K)<2 \delta / 3$. Let $j$ be the unique element of $\mathbf{N}$ such that $y \in \mathcal{2}_{j, \delta}$. Since $\mathcal{Q}_{j, \delta} \subset \mathbf{B}_{\delta / 3}\left(x_{j, \delta}\right)$, we conclude that $\operatorname{dist}\left(x_{j, \delta}, K\right)<\delta$; hence $K$ intersects $\mathbf{B}_{\delta}\left(x_{j, \delta}\right)=B_{j, \delta}$, which means that $j \in \mathcal{G}_{\delta}$. This proves (7.11).

We now write

$$
\sum_{j \in \mathcal{G}_{\delta}} g_{j, \delta}(x)=h_{\delta} *\left(\sum_{j \in \mathcal{G}_{\delta}} \chi_{2_{j, \delta}}\right)(x) \quad \text { if } x \in \mathbf{R}^{n} .
$$

According to (7.11) we have $\sum_{j \in \mathcal{g}_{\delta}} \chi_{2_{j . \delta}}(y)=1$ if $\operatorname{dist}(y, K)<2 \delta / 3$. Recalling the definition of $h_{\delta}$, we obtain Lemma 7.3.

LEMMA 7.4. If $F \in \mathbf{L}_{p}\left(\mathbf{R}^{n}\right)$, then

$$
\lim _{\delta \downarrow 0} \sum_{j}\left\|F-[F]_{\mathcal{Q}_{j, \delta}}\right\|_{p, \mathbf{B}_{j . \delta}}^{p}=0 .
$$


As Lindberg notes [25, p. 66], Lemma 7.4 is easy if $F$ is a continuous function of compact support in $\mathbf{R}^{n}$, and follows for arbitrary $F \in \mathbf{L}_{p}\left(\mathbf{R}^{n}\right)$ by using the fact that continuous functions of compact support are dense in $\mathbf{L}_{p}\left(\mathbf{R}^{n}\right)$. The details will be omitted.

We now complete this paper by proving the implication (c) $\Rightarrow$ (a) of Theorem 2.1. Suppose that condition (c) of that theorem holds. We let $f \in \mathbf{L}_{p}(X)$ satisfy $L(D) f=0$ on int $X$, and define $f=0$ on $\mathbf{R}^{n} \backslash X$.

If $f$ is considered as a distribution on $\mathbf{R}^{n}$, its support is contained in the compact set $X \subset \mathbf{R}^{n}$. It follows that

$$
f=\delta * f=L(D)(E * f)=E *(L(D) f) .
$$

We conclude from Lemmas 5.1 and 7.3 that, for each $\delta>0$, we have

$$
\sum_{j \in \mathscr{g}_{\delta}} \mathfrak{V}_{g_{j . \delta}}(f)=f
$$

where $\mathscr{q}_{\delta}$ is the set of all $j \in \mathbf{N}$ such that $B_{j, \delta}$ intersects $X$.

If $\delta>0$ and $j \in \mathbf{N}$, we conclude from Lemmas 5.3 and Lemma 7.2 (b) that

$$
\left\|\mathscr{V}_{g_{j, \delta}}(f)\right\|_{p, 2 B_{j, \delta}} \leqslant C_{1}\left\|f-[f]_{Q_{j, \delta}}\right\|_{p, B_{j, \delta}},
$$

where $C_{1}=C_{2} 2^{-n} n^{-n / 2} \sum_{|\alpha| \leqslant m} M_{|\alpha|}$ and $C_{2}$ denotes the constant $C$ of Lemma 5.3. We will let $C_{3}$ denote the constant $C(Z)$ of Lemma 7.1.

We now claim that, for each $\delta$ satisfying $0<\delta<\rho$, there exists a sequence of distributions $\left\{W_{j, \delta}\right\}_{j \in \mathbf{N}}$ in $\mathcal{E}^{\prime}\left(\mathbf{R}^{n}\right)$ with the following properties: for each $j \in \mathbf{N}$ we have supp $W_{j, \delta} \subset B_{j, \delta} \backslash X,\left\langle g_{j, \delta} L(D) f-W_{j, \delta}, \bar{I}\right\rangle=0$ if $I \in \mathscr{P}_{0} \cup \cdots \cup \mathscr{P}_{m-1}$, and (7.14) $E * W_{j, \delta} \in \mathbf{L}_{p}\left(\mathbf{R}^{n}\right.$, loc $)$ and $\left\|E * W_{j, \delta}\right\|_{p, 2 B_{j, \delta}} \leqslant \zeta \| f-[f]_{2_{j, \delta} \|_{p, B_{j, \delta}}}$, where $\zeta$ is a constant depending on $\eta$. Assuming for the moment that this claim has been proved, we let $0<\delta<\rho$, define $w_{j, \delta}=E * W_{j, \delta}$ for $j \in \mathbf{N}$, and conclude that

$$
\begin{aligned}
\left\|f-\sum_{j \in \mathcal{G}_{\delta}} w_{j, \delta}\right\|_{p, \mathbf{R}^{n}}^{p} & =\left\|\sum_{j \in \mathcal{G}_{\delta}}\left[\mathscr{V}_{g_{j, \delta}}(f)-w_{j, \delta}\right]\right\|_{p, \mathbf{R}^{n}}^{p} \\
& \leqslant C_{3} \sum_{j}\left\|\mathcal{V}_{g_{j, \delta}}(f)-w_{j, \delta}\right\|_{p, 2 B_{j, \delta}}^{p} \\
& \leqslant 2^{p / q} C_{3} \sum_{j}\left(\left\|\mathcal{V}_{g_{j, \delta}}(f)\right\|_{p, 2 B_{j, \delta}}^{p}+\left\|w_{j, \delta}\right\|_{p, 2 B_{j, \delta}}^{p}\right) \\
& \leqslant 2^{p / q} C_{3} \sum_{j}\left(C_{\mathrm{P}}^{p}+\zeta^{p}\right)\left\|f-[f]_{\mathcal{Q}_{j, \delta}}\right\|_{p, B_{j, \delta}}^{p},
\end{aligned}
$$

where the equality follows from (7.12), the first inequality follows from Lemma 7.1, the second inequality follows from (3.2), and the third inequality follows from (7.13) and (7.14). In view of Lemma 7.4 the last sum approaches zero as $\delta \downarrow 0$, which proves condition (a) of Theorem 2.1.

We now prove the claim of the preceding paragraph. In the proof we let $\delta$ be fixed with $0<\delta<\rho$; we write $B_{j}=B_{j, \delta}, \mathscr{Q}_{j}=\mathscr{2}_{j, \delta}$ and $g_{j}=g_{j, \delta}$. We will use induction on $\nu$ to prove the following assertion for $\nu \in\{0,1, \ldots, m-1\}$. 
$\Pi_{\nu}$ : There exists a sequence $\left\{W_{j}^{(\nu)}\right\}_{j \in \mathbf{N}}$ in $\mathcal{G}^{\prime}\left(\mathbf{R}^{n}\right)$ such that, for each $j \in \mathbf{N}$, we have $\operatorname{supp} W_{j}^{(\nu)} \subset B_{j} \backslash X,\left\langle g_{j} L(D) f-W_{j}^{(\nu)}, \bar{I}\right\rangle=0$ if $I \in \mathscr{P}_{0} \cup \cdots \cup \mathscr{P}_{\nu}, E * W_{j}^{(\nu)} \in$ $\mathbf{L}_{p}\left(\mathbf{R}^{n}\right.$, loc $)$ and $\left\|E * W_{j}^{(\nu)}\right\|_{p, 2 B_{j}} \leqslant \zeta_{\nu}\left\|f-[f]_{\mathscr{Q},}\right\|_{p, B_{j}}$.

Here the constants $\zeta_{\nu}$ are defined inductively by the equations $\zeta_{0}=2 \eta C_{1}$ and $\zeta_{\nu}=2 \eta C_{1}+(2 \eta+1) \zeta_{\nu-1}$ if $1 \leqslant \nu \leqslant m-1$. Since $\Pi_{m-1}$ implies the claim, with $\zeta=\zeta_{m-1}$ and $W_{j, \delta}=W_{j}^{(m-1)}$ for each $j \in \mathbf{N}$, this will complete the proof of Theorem 2.1.

To prove $\Pi_{0}$, we fix $j \in \mathbf{N}$. We note that $\operatorname{supp}\left[g_{j} L(D) f\right]$ is a compact subset of $B_{j} \backslash$ int $X$. We conclude that

$$
\begin{aligned}
\left|\left\langle g_{j} L(D) f, 1\right\rangle\right| & \leqslant \gamma_{p, 1}\left(B_{j} \backslash \operatorname{int} X, 2 B_{j}\right)\left\|\mho_{g_{j}}(f)\right\|_{p, 2 B_{j}} \\
& \leqslant \eta C_{1} \gamma_{p, 1}\left(B_{j} \backslash X, 2 B_{j}\right)\left\|f-[f]_{Q_{j}}\right\|_{p, B_{j}},
\end{aligned}
$$

where the first inequality follows from the definition of $\gamma_{p, 1}\left(B_{j} \backslash\right.$ int $\left.X, 2 B_{j}\right)$, and the second inequality follows from (7.13) and condition (c) of Theorem 2.1. From this estimate and the definition of $\gamma_{p, 1}\left(B_{j} \backslash X, 2 B_{j}\right)$ we deduce that there exists a distribution $W_{j}^{(0)} \in \mathcal{E}^{\prime}\left(\mathbf{R}^{n}\right)$ such that $\operatorname{supp} W_{j}^{(0)} \subset B_{j} \backslash X, \quad E * W_{j}^{(0)} \in \mathbf{L}_{p}\left(\mathbf{R}^{n}\right.$, loc $)$ and $\left\|E * W_{j}^{(0)}\right\|_{p, 2 B_{j}} \leqslant 2 \eta C_{1}\left\|f-[f]_{2,}\right\|_{p, B_{i}}$, and $\left\langle W_{j}^{(0)}, 1\right\rangle=\left\langle g_{j} L(D) f, 1\right\rangle$. This proves $\Pi_{0}$.

Now let $\nu \in\{1, \ldots, m-1\}$ be fixed, and suppose $\Pi_{\nu-1}$ has been proved. We let $j \in \mathbf{N}$ be fixed. If $W_{j}^{(\nu-1)} \in \mathcal{E}^{\prime}\left(\mathbf{R}^{n}\right)$ is the distribution given by $\Pi_{\nu-1}$, we define $S=g_{j} L(D) f-W_{j}^{(\nu-1)}$. Then the hypothesis $\Pi_{\nu-1}$ and (7.13) yield

$$
\langle S, \bar{I}\rangle=0 \quad \text { if } I \in \mathscr{P}_{0} \cup \cdots \cup \mathscr{P}_{\nu-1}
$$

and

$$
\begin{aligned}
\|E * S\|_{p, 2 B_{j}} & \leqslant\left\|\mathcal{V}_{g_{j}}(f)\right\|_{p, 2 B_{j}}+\left\|E * W_{j}^{(\nu-1)}\right\|_{p, 2 B_{j}} \\
& \leqslant\left(C_{1}+\zeta_{\nu-1}\right)\left\|f-[f]_{Q_{j}}\right\|_{p, B_{j}}{ }^{\circ}
\end{aligned}
$$

We now define $H \in \mathscr{P}_{\nu}$ by $H=\Sigma_{|\alpha|=\nu}\left\langle S, Y_{\alpha}\right\rangle Y_{\alpha}$ and note that

$$
\langle S, \bar{I}\rangle=\{H, I\} \quad \text { if } I \in \mathscr{P}_{\nu} \text {. }
$$

We distinguish two cases.

Case 1. $H=0$. In this case it follows from (7.17) that $\langle S, \bar{I}\rangle=0$ for $I \in \mathscr{P}_{\nu}$. We conclude that $\Pi_{\nu}$ holds with $W_{j}^{(\nu)}=W_{j}^{(\nu-1)}$ for $j \in \mathbf{N}$.

Case 2. $H \neq 0$. In this case we note that (7.17) implies

$$
\langle S, \bar{I}\rangle=0 \quad \text { if } I \in \mathscr{P}_{\nu} \text { and }\{H, I\}=0 .
$$

We now obtain

$$
\begin{aligned}
|\langle S, \bar{H}\rangle| & \leqslant \gamma_{p, H}\left(B_{j} \backslash \text { int } X, 2 B_{j}\right)\|E * S\|_{p, 2 B_{j}} \\
& \leqslant \eta \gamma_{p, H}\left(B_{j} \backslash X, 2 B_{j}\right)\left(C_{1}+\zeta_{\nu-1}\right)\left\|f-[f]_{\mathcal{Q}_{j}}\right\|_{p, B_{j}},
\end{aligned}
$$

where the first inequality follows from (7.15), (7.18) and the definition of $\gamma_{p, H}\left(B_{j} \backslash\right.$ int $\left.X, 2 B_{j}\right)$, and the second inequality follows from (7.16) and condition (c) of Theorem 2.1. From this estimate and the definition of $\gamma_{p, H}\left(B_{j} \backslash X, 2 B_{j}\right)$ we deduce 
that there exists a distribution $W^{\prime} \in \mathcal{E}^{\prime}\left(\mathbf{R}^{n}\right)$ such that supp $W^{\prime} \subset B_{j} \backslash X,\left\langle W^{\prime}, \bar{I}\right\rangle=0$ if $I \in \mathscr{P}_{0} \cup \cdots \cup \mathscr{P}_{\nu-1},\left\langle W^{\prime}, \bar{I}\right\rangle=0$ if $I \in \mathscr{P}_{\nu}$ and $\{H, I\}=0, E * W^{\prime} \in \mathbf{L}_{p}\left(\mathbf{R}^{n}\right.$, loc $)$ and $\left\|E * W^{\prime}\right\|_{p, 2 B,} \leqslant 2 \eta\left(C_{1}+\zeta_{\nu-1}\right)\left\|f-[f]_{2}\right\|_{p, B}$, and $\left\langle W^{\prime}, \bar{H}\right\rangle=\langle S, \bar{H}\rangle$. From these properties and (7.18) we see that $\Pi_{\nu}$ holds with $W_{j}^{(\nu)}=W_{j}^{(\nu-1)}+W^{\prime}$ for each $j \in \mathbf{N}$.

Appendix (added in proof). We give here an alternate definition of the capacity $\gamma_{p, H^{*}}$. One can prove the following result concerning the behavior of the potential $E * T(x)$ for $|x|$ large (compare [10]). If $l \in\{0, \ldots, m-1\}$ and $T \in \mathcal{E}^{\prime}\left(\mathbf{R}^{n}\right)$ are fixed, then there is one and only one sequence $H_{0} \in \mathscr{P}_{0}, \ldots, H_{l} \in \mathscr{P}_{l}$ such that $E * T(x)$ $=\sum_{k=0}^{l} H_{k}(D) E(x)+O\left(|x|^{m-n-l-1}\right)$ as $|x| \rightarrow \infty$. Moreover, for each $k \in\{0, \ldots, l\}$ we have

$$
H_{k}=(-1)^{k} \sum_{|\alpha|=k}\left\langle T, Y_{\alpha}\right\rangle Y_{\alpha},
$$

and hence $\left\{H_{k}, I\right\}=(-1)^{k}\langle T, \bar{I}\rangle$ if $I \in \mathscr{P}_{k}$. Note that if $l \in\{0, \ldots, m-1\}$ and $T \in \mathcal{E}^{\prime}\left(\mathbf{R}^{n}\right)$, then in the terminology of this result (2.6) states that $H_{k}=0$ for $0 \leqslant k \leqslant l-1$, (2.7) states that $H_{l}$ is a scalar multiple of $H$, and $|\langle T, \bar{H}\rangle|=$ $\left|\left\{H_{k}, H\right\}\right|$. As a corollary we obtain the following theorem, which gives an alternate definition of $\gamma_{p, H}$.

Let $l \in\{0, \ldots, m-1\}$ and $H \in \mathscr{P}_{\curlywedge} \backslash\{0\}$, and let $A$ be a subset of the open set $\Omega \subset \mathbf{R}^{n}$. Let $\Re$ denote the set of all distributions $T \in \mathcal{G}^{\prime}\left(\mathbf{R}^{n}\right)$ such that $\operatorname{supp} T \subset A$ and $E * T(x)=H(D) E(x)+O\left(|x|^{m-n-l-1}\right)$ as $|x| \rightarrow \infty$. Then $\gamma_{p, H}(A, \Omega)=$ $\{H, H\} / \inf _{T \in \mathfrak{R}}\|E * T\|_{p, \Omega}$.

If $A$ is empty, then $\mathscr{T}$ is empty and the right side of the last equation is understood to be zero. If $A$ is nonempty, then $\mathcal{K}$ is also: if $a \in A$, and $\boldsymbol{\delta}_{a}$ denotes the Dirac measure at $a$, then we see that $H(D) \boldsymbol{\delta}_{a} \in \mathcal{K}$ by applying Lemma 3.7 to the distribution $H(D)\left(\boldsymbol{\delta}_{a}-\boldsymbol{\delta}\right)$.

\section{REFERENCES}

1. D. R. Adams and J. C. Polking, The equivalence of two definitions of capacity, Proc. Amer. Math. Soc. 37 (1973), 529-534.

2. I. Babuška, Stability of the domain with respect to the fundamental problems in the theory of partial differential equations, mainly in connection with the theory of elasticity. I, II, Czechoslovak Math. J. 11 (86) (1961), 76-105, 165-203. (Russian)

3. T. Bagby, Quasi topologies and rational approximation, J. Funct. Anal. 10 (1972), 259-268.

4. V. I. Burenkov, On the approximation of functions in the space $W_{p}^{r}(\Omega)$ by functions with compact support for an arbitrary open set $\Omega$, Trudy Mat. Inst. Steklov. 131 (1974), 51-63; English transl., Proc. Steklov Inst. Math. 131 (1974), 53-66.

5. A. P. Calderón, Lecture notes on pseudo differential operators and elliptic boundary value problems, Cursos de Matematica 1, Instituto Argentino de Matematica, Buenos Aires, 1976.

6. L. Carleson, Mergelyan's theorem on uniform polynomial approximation, Math. Scand. 15 (1964), 167-175.

7. C. Fernström and J. C. Polking, Bounded point evaluations and approximation in $L^{p}$ by solutions of elliptic partial differential equations, J. Funct. Anal. 28 (1978), 1-20.

8. T. W. Gamelin, Uniform algebras, Prentice-Hall, Englewood Cliffs, N. J., 1969.

9. R. Harvey and J. C. Polking, A notion of capacity that characterizes removable singularities, Trans. Amer. Math. Soc. 169 (1972), 183-195.

10. __ A Laurent expansion for solutions to elliptic equations, Trans. Amer. Math. Soc. 180 (1973), 407-413. 
11. V. P. Havin, Approximation in the mean by analytic functions, Dokl. Akad. Nauk SSSR 178 (1968), 1025-1028; English transl., Soviet Math. Dokl. 9 (1968), 245-248.

12. L. I. Hedberg, Approximation in the mean by analytic functions, Trans. Amer. Math. Soc. 163 (1972), 157-171.

13. Non-linear potentials and approximation in the mean by analytic functions, Math. Z. 129 (1972), 299-319.

14. __ Approximation in the mean by solutions of elliptic equations, Duke Math. J. 40 (1973), 9-16.

15. __ Removable singularities and condenser capacities, Ark. Mat. 12 (1974), 181-201.

16. Two approximation problems in function spaces, Ark. Mat. 16 (1978), 51-81.

17. Spectral synthesis in Sobolev spaces, and uniqueness of solutions of the Dirichlet problem, Acta Math. 147 (1981), 237-264.

18. L. I. Hedberg and T. H. Wolff, Thin sets in nonlinear potential theory (to appear).

19. L. Hörmander, Linear partial differential operators, Academic Press, New York, 1963.

20. F. John, Plane waves and spherical means applied to partial differential equations, Interscience, New York, 1955.

21. M. V. Keldyš, On the solubility and stability of the Dirichlet problem, Uspehi Mat. Nauk 8 (1941), 171-231; English transl., Amer. Math. Soc. Transl. (2) 51 (1966), 1-73.

22. P. Lax, A stability theory of abstract differential equations and its applications to the study of local behaviors of solutions of elliptic equations, Comment. Pure Appl. Math. 9 (1956), 747-766.

23. N. S. Landkof, Foundations of modern potential theory, "Nauka”, Moscow, 1966; English transl., Springer-Verlag, New York, 1972.

24. P. Lindberg, $L^{P}$-approximation by analytic functions in an open region, Uppsala Univ. Dept. of Math. Report No. 1977:7.

25. $61-68$. , A constructive method for $L^{p}$-approximation by analytic functions, Ark. Mat. 20 (1982),

26. B. Malgrange, Existence et approximation des solutions des équations aux deriveés partielles et des équations de convolutions, Ann. Inst. Fourier (Grenoble) 6 (1955-1956), 271-355.

27. V. G. Maz'ja, On ( $p, l)$-capacity, imbedding theorems, and the spectrum of a self adjoint elliptic operator, Izv. Akad. Nauk SSSR Ser. Math. 37 (1973), 356-385; English transl., Math. USSR-Izv. 7 (1973), 357-387.

28. S. N. Mergeljan, Uniform approximations to functions of a complex variable, Uspehi Mat. Nauk 7 (1952), 31-122; English transl., Amer. Math. Soc. Transl. (1) 101 (1954).

29. A. G. O'Farrell, Metaharmonic approximation in Lipschitz norms, Proc. Roy. Irish Acad. Sect. A 75 (1975), 317-330.

30. J. C. Polking, Approximation in $L^{p}$ by solutions of elliptic partial differential equations, Amer. J. Math. 94 (1972), 1231-1244.

31. J. C. Polking, A Leibniz formula for some differentiation operators of fractional order, Indiana Univ. Math. J. 21 (1972), 1019-1029.

32. C. Runge, Zur Theorie der eindeutigen analytischer Funktionen, Acta Math. 6 (1885), 229-244.

33. Ė. M. Saak, $A$ capacity condition for a domain with a stable Dirichlet problem for higher order elliptic equations, Mat. Sb. 100(142) (1976), 201-209; English transl., Math. USSR Sb. 29 (1976), 177-185.

34. G. E. Šilov, Generalized functions and partial differential equations, Gordon \& Breach, New York, 1968.

35. E. M. Stein, Singular integrals and differentiability properties of functions, Princeton Univ. Press, Princeton, N. J., 1970.

36. A. G. Vituškin, Analytic capacity of sets and problems in approximation theory, Uspehi Mat. Nauk 22 (1967), 141-199; English transl., Russian Math. Surveys 22 (1967), 139-200.

37. B. Weinstock, Uniform approximation by solutions of elliptic equations, Proc. Amer. Math. Soc. 41 (1973), 513-517.

Department of Mathematics, Swain Hall-East, Indiana University, Bloomington, Indiana 47405 